\title{
Quantifying fluorescent glycan uptake to elucidate strain-level variability in foraging behaviors of rumen bacteria
}

Leeann Klassen ${ }^{1,2+}$, Greta Reintjes ${ }^{1,3 \dagger}$, Jeffrey P. Tingley ${ }^{1}$, Darryl R. Jones ${ }^{1}$, Jan-Hendrik Hehemann ${ }^{3,4}$, Adam D. Smith ${ }^{1}$, Timothy D. Schwinghamer ${ }^{1}$, Carol Arnosti ${ }^{5}$, Long Jin ${ }^{1}$, Trevor W. Alexander ${ }^{1}$, Carolyn Amundsen ${ }^{1}$, Dallas Thomas', Rudolf Amann ${ }^{3}$, Tim A. McAllister ${ }^{1}$ and D. Wade Abbott ${ }^{1,2^{*}}$

\begin{abstract}
Gut microbiomes, such as the microbial community that colonizes the rumen, have vast catabolic potential and play a vital role in host health and nutrition. By expanding our understanding of metabolic pathways in these ecosystems, we will garner foundational information for manipulating microbiome structure and function to influence host physiology. Currently, our knowledge of metabolic pathways relies heavily on inferences derived from metagenomics or culturing bacteria in vitro. However, novel approaches targeting specific cell physiologies can illuminate the functional potential encoded within microbial (meta)genomes to provide accurate assessments of metabolic abilities. Using fluorescently labeled polysaccharides, we visualized carbohydrate metabolism performed by single bacterial cells in a complex rumen sample, enabling a rapid assessment of their metabolic phenotype. Specifically, we identified bovine-adapted strains of Bacteroides thetaiotaomicron that metabolized yeast mannan in the rumen microbiome ex vivo and discerned the mechanistic differences between two distinct carbohydrate foraging behaviors, referred to as "medium grower" and "high grower." Using comparative wholegenome sequencing, RNA-seq, and carbohydrate-active enzyme fingerprinting, we could elucidate the strain-level variability in carbohydrate utilization systems of the two foraging behaviors to help predict individual strategies of nutrient acquisition. Here, we present a multi-faceted study using complimentary next-generation physiology and "omics" approaches to characterize microbial adaptation to a prebiotic in the rumen ecosystem.
\end{abstract}

Keywords: Microbiome, Carbohydrate, Rumen, Glycoside hydrolase, Fluorescent polysaccharides, Yeast mannan, Bacteroides

\section{Background}

Ruminants have evolved foregut digestive systems specialized in the bioconversion of recalcitrant, complex carbohydrates into energy. These catabolic processes rely on a core bacterial community composed predominantly

\footnotetext{
* Correspondence: wade.abbott@canada.ca

${ }^{\dagger}$ Leeann Klassen and Greta Reintjes contributed equally to this work.

'Lethbridge Research and Development Centre, Agriculture and Agri-Food

Canada, 5403-1st Avenue South, Lethbridge, Alberta T1J 4B1, Canada

${ }^{2}$ Department of Biological Sciences, University of Lethbridge, Lethbridge, Alberta T1K 3M4, Canada

Full list of author information is available at the end of the article
}

of the genera Prevotella, Butyrivibrio, Fibrobacter, and Ruminococcus, families Lachnospiraceae and Ruminococcaceae, and orders Bacteroidales and Clostridiales [1, 2]. The rumen microbiome is estimated to contain 69,000 carbohydrate-active enzyme (CAZyme) genes [3] that encode extensive catalytic activities. Despite this vast genetic repertoire and catalytic potential, the microbial conversion of plant fiber to host-accessible metabolites in the rumen is suboptimal and could be improved [4, 5]. For example, supplementation with direct fed microorganisms, such as Bacteroides spp., or prebiotic 
carbohydrates that modify the rumen microbiome to enhance feed conversion may help address the emerging challenges associated with sustainable production of food animals [6].

In diverse animal symbioses and environmental ecosystems, Bacteroides spp. and other members of Bacteroidetes are thought to play central roles in glycan digestion because they encode highly specialized carbohydrate metabolic systems called polysaccharide utilization loci (PULs) $[7,8]$. The first described PUL was the starch utilization system of Bacteroides thetaiotaomicron (B. theta: BtVPI-5482) [9], and since its description, PULs that metabolize glycans with unique chemistries have been found in diverse ecosystems $[8,10-12]$. PULs are distinguished by the presence of a TonB-dependent transporter coupled to a surface glycanbinding protein, known as the SusC/D-like complex, and other associated proteins that modify or bind the target glycan. These gene products function together in an orchestrated cascade to transport oligosaccharides into the periplasm where monosaccharides are released from polymeric substrates and used for primary metabolism. PULs can operate through a "distributive" mechanism, which releases products [13] to the microbial community or a "selfish" mechanism [14], which limits product loss by confining substrate depolymerization within the cell $[8,15]$. Recently, PUL-prediction [16] and whole-PUL characterization $[14,17]$ have become common approaches for the discovery of new CAZyme families and catalytic activities at the species [18] and strain levels [19, 20]. The most common enzymes encoded within PULs are glycoside hydrolases (GHs), which cleave glycosidic bonds by acidbase catalysis [21]. GHs are divided into sequence-related families that display conserved folds, mechanisms, and catalytic residues. However, these features are not necessarily representative of function as many different $\mathrm{GH}$ families are polyspecific [22].

In addition to microorganisms that improve the efficiency of digestion, prebiotic glycans, such as yeast $\alpha-$ mannan (YM) and its derivative oligosaccharides (i.e., $\alpha-$ mannanoligosaccharides), are known to provide beneficial physiological outcomes to animals, such as cattle and pigs [23-25]. Prebiotics can enhance feed digestion and cattle health by becoming selective nutrients for symbiotic gut bacteria, such as Bacteroides spp. The digestion of YM requires a collection of CAZymes targeting distinct linkages using different modes of activity, including $\alpha$-mannanases and $\alpha$-mannosidases [15]. CAZymes that possess these activities are commonly found in family GH38, GH76, GH92, GH99, and GH125 $[14,26,27]$, and correspondingly, these enzymes are present in PULs that target YM (i.e., MAN-PULs).

YM-specific CAZymes and PULs are widely distributed in Bacteroidetes; however, individual species differ in their abilities to consume $\alpha$-mannans depending on the structural complexity of the substrate [14]. For example, Bacteroides xylanisolvens NLAE-zl isolated from pigs reared on a diet infused with distillers' grains could only metabolize debranched YM [14]. The pathway responsible for YM catabolism in these strains (i.e., MANPUL1) was encoded on a transposable element, suggesting that aspects of YM metabolism can be exchanged between strains [14]. This finding is consistent with reports of specialized metabolic abilities being transferred to intestinal Bacteroides spp. from species that occupy ecologically distinct habitats $[19,20,28]$, facilitating their persistence within highly competitive ecosystems and adaption to spatially and culturally diversified diets.

Although major advances have been made in understanding the diversity of metabolic potential in symbiotic bacteria and the mechanisms of prebiotic utilization, establishing stable engineered microbiomes in complex ecosystems, such as the rumen, will require more detailed knowledge of the competitive and complementary processes that drive metabolic phenotypes at the strain level. To achieve this, "next-generation physiology"based [29] approaches that identify metabolic potentials of individual bacteria, thereby providing critical insights of cellular functions and assigning cellular phenotypes, must be developed. One such approach is fluorescently labeled polysaccharides (FLA-PS). FLA-PS were initially developed to demonstrate selfish uptake of marine polysaccharides in marine Bacteroidetes [30] and have also been applied to the gut bacterium BtVPI-5482 to confirm that YM metabolism also occurs through a selfish mechanism [31]. Fluorescent glucose analogs have been recently used to study glucose uptake by rumen bacteria [32]; however, use of fluorescent polysaccharides in the rumen has been limited until now.

Here, for the first time, we apply FLA-PS as a nextgeneration physiology approach to directly visualize YM metabolism by single cells in a complex rumen community and subsequently classify populations of cells using fluorescence in situ hybridization (FISH). We combine this analysis with a multi-tiered study of the evolution and function of YM metabolism in bovine-adapted $B$. theta strains $\left(B t^{\mathrm{Bov}}\right)$, which adopt one of two dichotomous growth phenotypes, referred to as "High Grower" (HG) or "Medium Grower" (MG), based on the optical density of cultures after $24 \mathrm{~h}$. Despite displaying distinct growth profiles, the genetic, transcriptomic, or biochemical factors that contributed to the differential growth phenotypes of these strains remained to be defined. Using genomics, transcriptomics, and CAZyme fingerprinting, multiple MAN-PUL architectures were identified in this study that are consistent with reports for human-associated BtVPI-5482 [14] and key differences in the YM utilization systems between MGs and HGs were revealed. To define the mechanisms that contribute 
to these growth phenotypes, we present a new quantitative application of FLA-PS, which we believe has farreaching implications for elucidating differences in substrate utilization of individual cells within complex microbial communities.

\section{Results}

\section{Ex vivo visualization of YM-metabolizing taxa within the} rumen community

To assess the capability of rumen microbiota to metabolize YM, extracted rumen samples were incubated with FLA-YM and visualized on feed particles and in solution (Fig. 1a, b). The total cell density in $100 \mu \mathrm{m}$ pre-filtered rumen fluid, as determined by enumerating DAPI-stained cells, was $2.98 \times 10^{8} \pm 6.02 \times 10^{7}$ cells $\mathrm{ml}^{-1}$ (Fig. 1c). In these complex communities, on average $6.1 \% \pm 0.5 \%$ of cells showed uptake of FLA-YM $(0 \%$ after $15 \mathrm{~min}, 6 \%$ after $3 \mathrm{~h}, 7 \%$ after 1 day, $6 \%$ after 3 days). Fluorescence in situ hybridization (FISH) using the CF968 probe [33] specific for the phylum Bacteroidetes showed that $2.9 \pm 0.5 \%$ of the cells showing FLAYM uptake were members of the Bacteroidetes. In total, Bacteroidetes made up $34.8 \% \pm 6.8 \%$ of the rumen bacterial community and only a fraction of these $(\sim 3 \%)$ showed uptake of FLA-YM (Fig. 1c). The microbial community composition of these rumen samples was determined by $16 \mathrm{~S}$ rRNA metagenomics sequencing. The community was dominated by Bacteroidetes, specifically the genus Prevotella 1, which demonstrated that YM metabolism has penetrated distantly related members of the phylum (Fig. 1d).

\section{Isolation and growth profiling of YM-utilizing $B t^{\text {Bov }}$ strains} Targeted isolation approaches were performed to selectively isolate bovine-adapted-bacteria that utilize Saccharomyces cerevisiae YM from enriched rumen and fecal communities. Single colonies were observed within $24 \mathrm{~h}$, with new colonies forming up to $96 \mathrm{~h}$. In total, 50 bacterial isolates were collected and each mannandegrading (MD) isolate was assigned a reference number (e.g., isolate $\# 8=\mathrm{MD} 8$ ). The majority of these isolates were identified by $16 \mathrm{~S}$ rRNA gene sequencing as strains of $B$. theta using the NCBI BLASTN database [34], and referred to as $B t^{\mathrm{Bov}}$ (Fig. 2a, Supplementary Table 1).

YM metabolism was confirmed for each MD strain by growth in liquid cultures using S. cerevisiae $\mathrm{YM}$ as the sole carbon source (Fig. 2b, Supplementary Fig. 1). Interestingly, based on their growth on S. cerevisiae YM, the $B t^{\mathrm{Bov}}$ isolates and BtVPI-5482 control strain were divided into two populations (Supplementary Table 2): "Medium Growers" (MGs; plateaued growth at $\mathrm{OD}_{600}$ 0.4 after 24 h) and "High Growers" (HGs; plateaued growth at $\mathrm{OD}_{600} \sim 0.7$ after $24 \mathrm{~h}$ ). Notably, this growth phenotype is substrate specific and does not extend to other substrates, such as mannose in which all strains have a similar growth curve (data not shown), and YM from Schizosaccharomyces pombe (S. pombe; Supplementary Fig. 1b). In addition, the $16 \mathrm{~S}$ rRNA gene topology of the $B t^{\mathrm{Bov}}$ isolates did not reveal a discernable relationship with the growth phenotype (Fig. 2a).

\section{Visualization and quantification of differential FLA-YM uptake by $B t^{B o v}$ isolates}

To determine if the rate of glycan uptake varied between the two growth types, representative strains, one from each growth population and BtVPI-5482 as a control, were incubated with FLA-YM. BtVPI-5482, MD33 ${ }_{\mathrm{MG}}$, and MD40 ${ }_{\mathrm{HG}}$ cells became fluorescent, whereas cells incubated with unlabeled YM did not (Fig. 2c). Phenotypic differences in FLA-YM uptake over time were determined by splitting cell populations by flow cytometric gating into FLA-positive and FLA-negative cells (Supplementary Fig. 2a). The total fluorescence intensity (rate of uptake) was significantly different between the representative strains

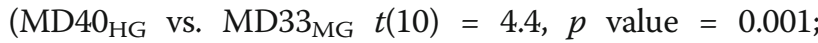

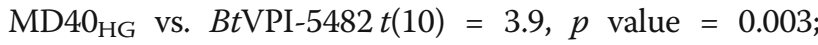
$B t$ VPI-5482 vs. MD33 ${ }_{\mathrm{MG}} t(10)=4.0, p$ value $\left.=0.002\right)($ Fig. $2 \mathrm{~d})$. The change in mean fluorescence of the three strains showed a similar temporal pattern, increasing from 0 to $120 \mathrm{~min}$, peaking at $120 \mathrm{~min}$, and declining from 120 to 1440 min (Fig. 2e). Although all three strains showed uptake of FLA-YM after $60 \mathrm{~min}$, MD33 $3_{\mathrm{MG}}$ had the lowest fluorescence intensity at each time point. $\mathrm{MD} 40_{\mathrm{HG}}$ displayed the highest fluorescence intensity, 2.7-fold higher than MD33 $3_{\text {MG. }}$ BtVPI-5482 displayed a fluorescence intensity between the two bovine strains for each time point, with a peak value 1.9-fold higher than MD33 ${ }_{\mathrm{MG}}$. Additionally, $\mathrm{MD} 40_{\mathrm{HG}}$ cells showed a more rapid uptake $(22 \%$ at $5 \mathrm{~min}$ ), $B t \mathrm{VPI}-5482$ cells showed an intermediate rate of uptake (9\% at $5 \mathrm{~min}$ ), and MD33 $3_{\mathrm{MG}}$ had the slowest uptake rate ( $2 \%$ at $5 \mathrm{~min}$ ) (Fig. 2e, Supplementary Fig. 2b).

To test if the phenotypic differences were inherited between generations, we measured the differences in FLAYM uptake with and without prior exposure to YM. The cultures continued to display the same phenotypic uptake patterns $\left(\mathrm{MD} 40_{\mathrm{HG}}\right.$ and $\mathrm{BtVPI}-5482$ higher uptake, MD33 $_{\text {MG }}$ low uptake, Fig. 3a-c). However, previous exposure to YM resulted in a heightened cellular response as indicated by more rapid rates of FLA-YM uptake relative to cultures previously grown on mannose-MM (Fig. $3 \mathrm{~b}-\mathrm{d})$. All cultures grown on mannose-MM reached a lower mean fluorescence and the temporal change in mean fluorescence was slower, with quantifiable uptake occurring only after 4 to $8 \mathrm{~h}$.

\section{Characterization of genotypes by PUL delineation}

Whole-genome sequencing and de novo assembly were used to identify genes involved in YM metabolism. 
a

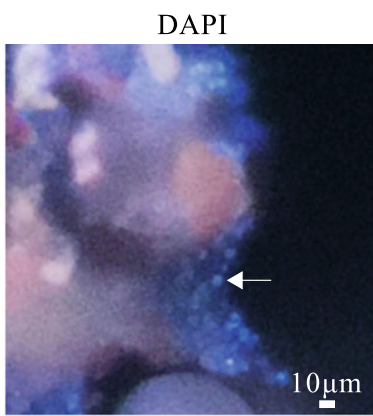

b

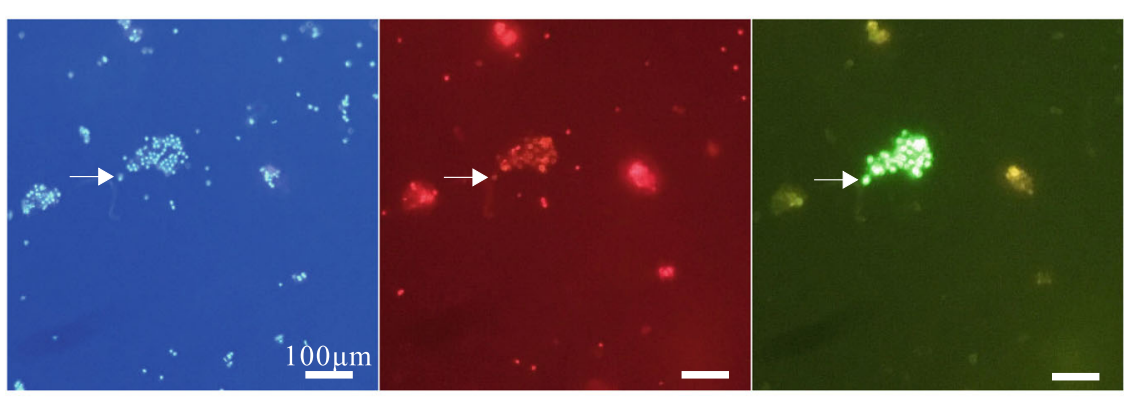

c

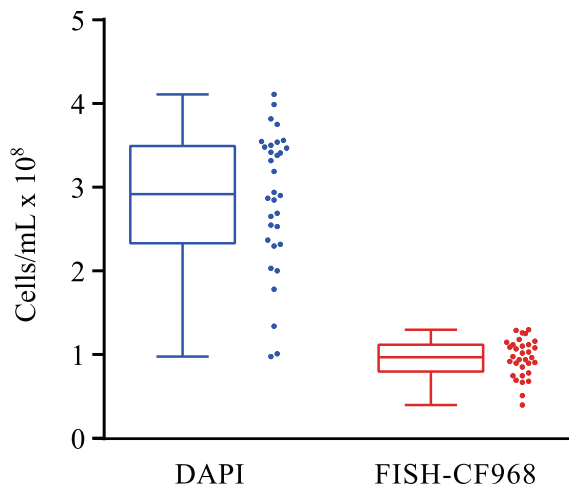

FISH-CF968

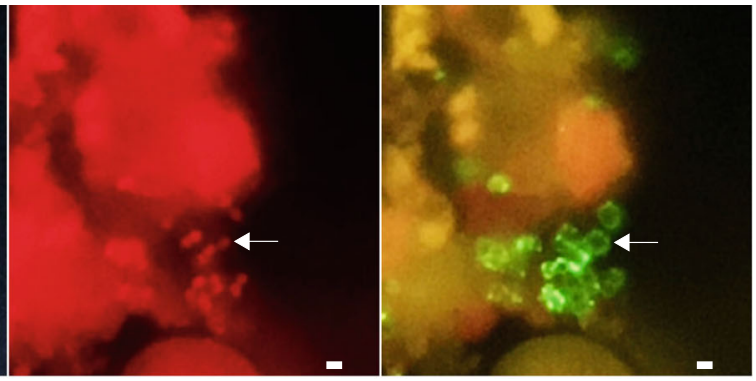

0.8

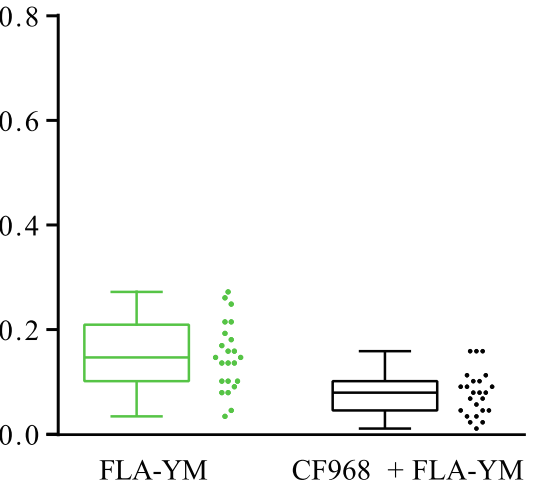

d Total bacterial community

Abundance (\%)

\begin{tabular}{cccccc}
0 & 20 & 40 & 60 & 80 & 100 \\
$L$ & 1 & $\mid$ & $\mid$ & $\mid$ & \\
\hline
\end{tabular}

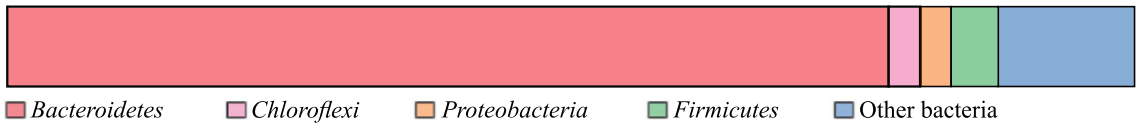

Bacteroidetes

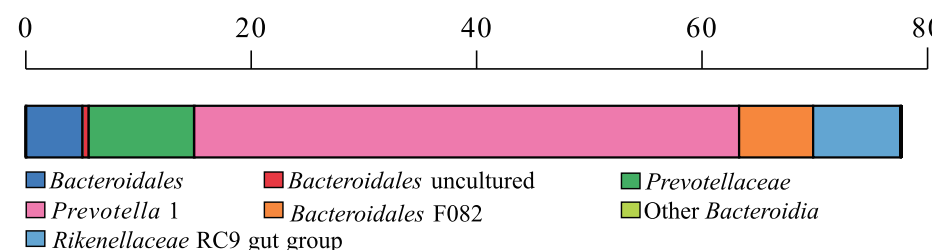

Fig. 1 YM utilization by $B t^{\text {Bov }}$ isolates. Images of a $100 \mu \mathrm{m}$ on food particles and $\mathbf{b} 10 \mu \mathrm{m}$ filtered rumen extract incubated with FLA-YM and stained with DAPI and Bacteroides-FISH probe (FISH-CF968). Cells were visualized by epifluorescence microscopy. c Counts of cells from FLA-YM incubated rumen extract stained with DAPI, FISH-CF968, FLA-YM, and both FISH-CF968 and FLA-YM. Mean \pm standard deviation shown. d $16 \mathrm{~S}$ rRNA metagenomics sequencing data of extracted rumen communities showing most prevalent phyla (left) and the distribution of Bacteroidetes spp. (right). $N=4$ 

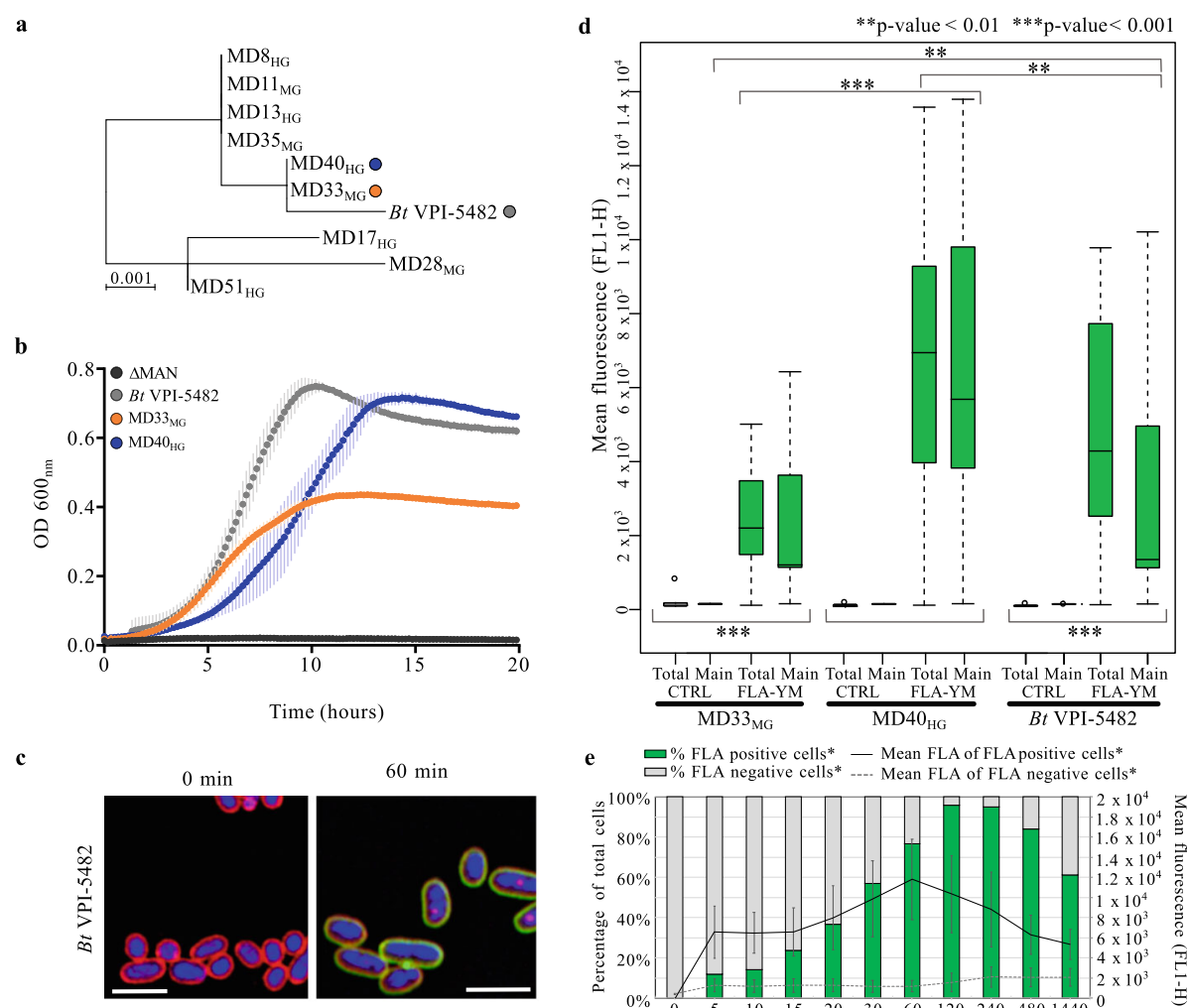

e

$\square$ FLA positive cells* — Mean FLA of FLA positive cells*
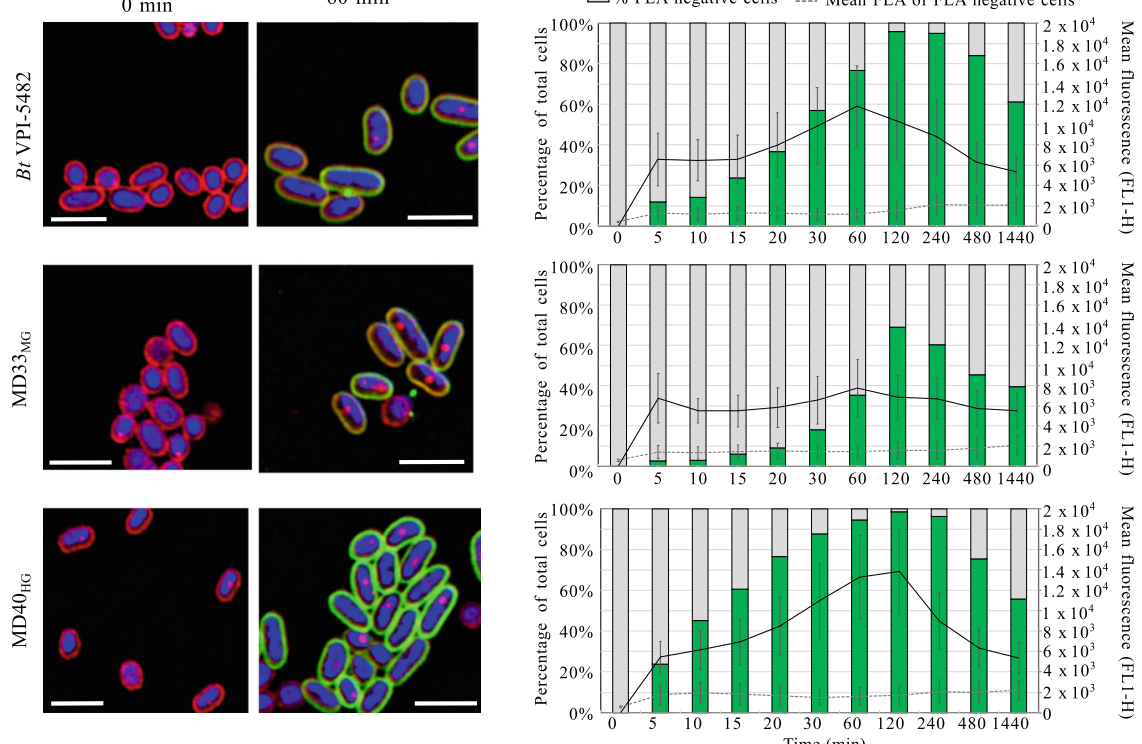

Fig. 2 Characterization of $B t^{B \circ v}$ isolates. a $16 S$ rRNA gene sequence comparison of $B t V P I-5482$ and $B t^{\text {Bov }}$ strains. Scale represents number of nucleotide changes across horizontal axis. b Growth profiles of BtVPI-5482, Bt $\triangle M$ MAN-PUL1/2/3, MD33 MG, and MD40 ${ }_{H G}$ grown on 0.5\% YM-MM. c SR-SIM of B. theta strains 0-min and 60-min post-incubation with FLA-YM. Cells co-stained with Nile Red and DAPI. d Mean fluorescence of bacterial cells cultured in FLA-YM or YM-MM (control). ${ }^{* *} p$ value $<0.01$; ${ }^{* *} p$ value $<0.001$; ns $p$ value $>0.05$. e Bars represent percentage of total cells showing an uptake of FLA-YM in cultures sampled over time. Green = increased FLA-YM uptake; grey = no uptake. Mean cell fluorescence represented by line graph; solid line = mean of cells with FLA-YM signal; dotted line = mean of cells showing no FLA-YM uptake

SPAdes [35] assembly output and average nucleotide identity based on BLAST+ (ANIb) [36] are shown in Supplementary Table 2. The ANIb results supported the $16 \mathrm{~S}$ rRNA gene sequence data, confirming that each isolate was a strain of $B$. theta. Furthermore, comparative genomics revealed that these strains have acquired unique CAZome repositories (Fig. 4a) and PUL updates (Supplementary Fig. 3 ); features that may assist with their colonization of the bovine gut and represents opportunities for developing bovine-adapted probiotics (Supplementary Discussion).
Reconstruction of the three YM-specific PULs and alignment with $B t$ VPI-5482 MAN-PULs determined that there was a high level of synteny among all strains in these pathways (Supplementary Fig. 4a). MAN-PUL2 and MAN-PUL3 were absolutely conserved, whereas MAN-PUL1, a PUL tailored for the consumption of mannan from S. pombe [14] (Supplementary Fig. 1b), was only present in BtVPI-5482, MD33 $3_{\mathrm{MG}}$, and

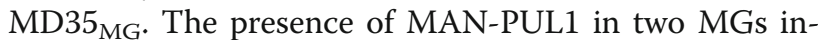
dicated this pathway was not responsible for the HG 


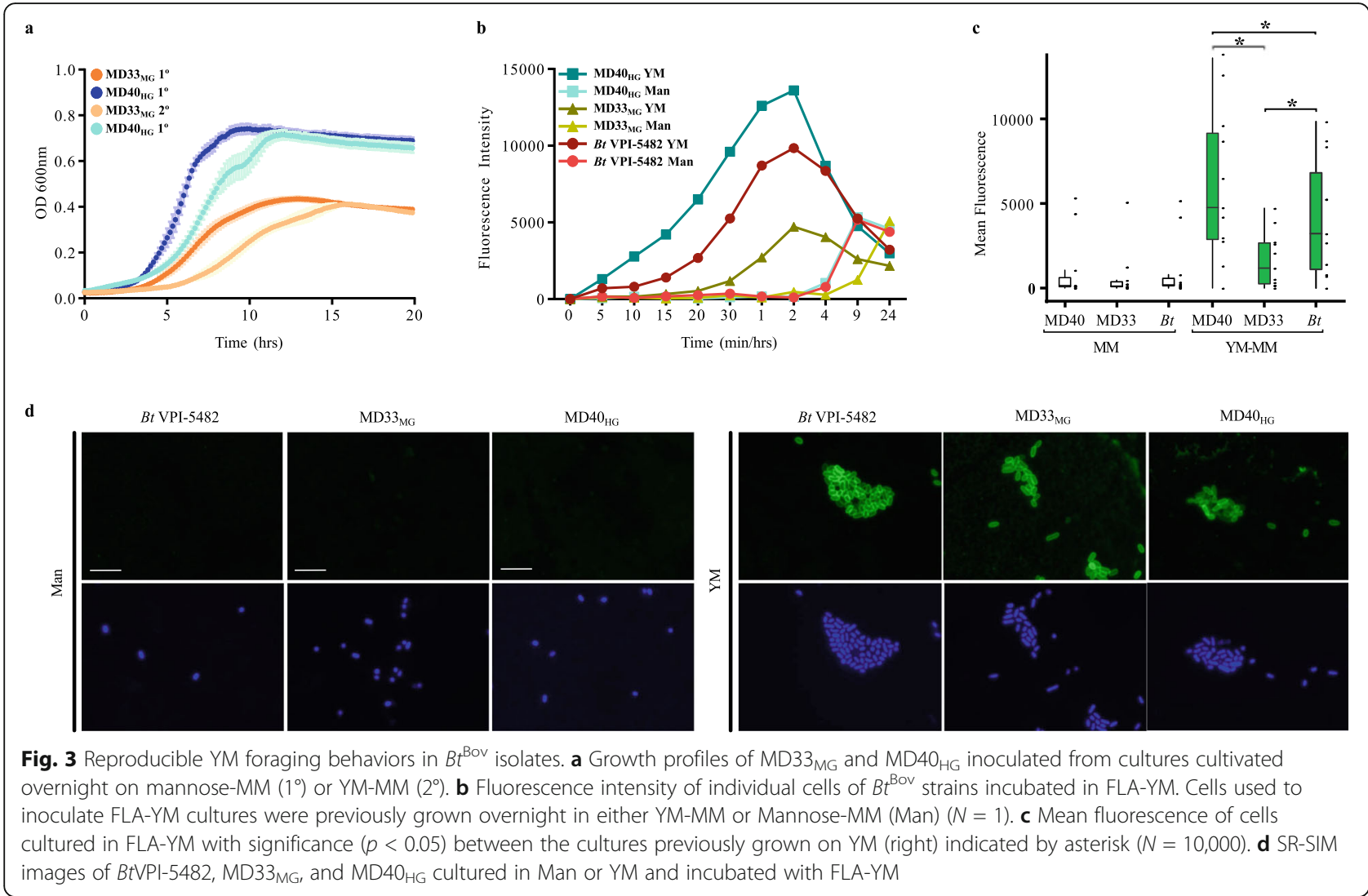

phenotype. The HMNG-PUL, which is specific for digestion of high mannose $\mathrm{N}$-glycans and not activated by YM in BtVPI-5482 [14], was also conserved in each of the $B t^{\mathrm{Bov}}$ genomes.

\section{CAZome fingerprinting}

To determine if there was amino acid sequence divergence within MAN-PULs, and potentially the function of homologous enzymes, polyspecific CAZyme families GH92 and GH76 were analyzed by SACCHARIS [37]. Enzyme sequences from GH92 and GH76 were embedded into phylogenetic trees comprised of all characterized enzyme sequences from each family (Supplementary Fig. 4b, c). Notably, every sequence within MAN-PUL1, MAN-PUL2, and MAN-PUL3 displayed the highest level of amino acid sequence conservation with its syntenic homolog. This suggested that each PUL is under strong selective pressure to function as an intact catabolic system. To determine if CAZyme sequences were conserved in other potential $\alpha$-mannandegrading PULs, a genome-wide approach (i.e., CAZome fingerprinting) was used [37]. Each isolate encoded between twenty-four and twenty-six GH92s and eight or nine GH76s (Fig. 4b, Supplementary Fig. 4b,c). Only $\mathrm{MD}_{17} 7_{\mathrm{HG}}$ and $\mathrm{MD} 51_{\mathrm{HG}}$ displayed identical conservation for GH76, whereas every GH92 tree was unique.
Topological differences were observed for other $\alpha$ mannan active enzyme families (e.g., GH38, GH99, and GH125), suggesting that despite the high level of functional conservation within the MAN-PULs, metabolic specialization in $\alpha$-mannan consumption between these strains may be encoded within orphan PULs [38]. Therefore, the contributions of two exogenous GH76s to the foraging behavior of HGs and MGs were investigated. BtGH76-MD40 is a surface-exposed GH76 inserted into PUL55 of HGs (Fig. 4b) and is active on intact $S$. cerevisiae and S. pombe YM [39]. BT_3782 is a periplasmic endo- $\alpha$-mannanase that generates small oligosaccharide products [14]. Addition of recombinant BtGH76-MD and BT_3782 to pure cultures of MD33 ${ }_{\mathrm{MG}}$ did not augment the MG growth phenotype (Supplementary Fig. 5), suggesting that acquisition of BtGH76-MD40 or augmented endo-mannanase activity were not responsible for the HG phenotype.

\section{Differences in YM import between phenotypes}

The differential transport kinetics of FLA-YM (Fig. 2ce) and absence of genetic differences in PUL structure between phenotypes (Supplementary Fig. 4a) suggested that glycan transport processes may be responsible for the MG and HG growth phenotypes. Alignment of the SusC-like amino acid sequences from MAN-PULs 1, 2, 


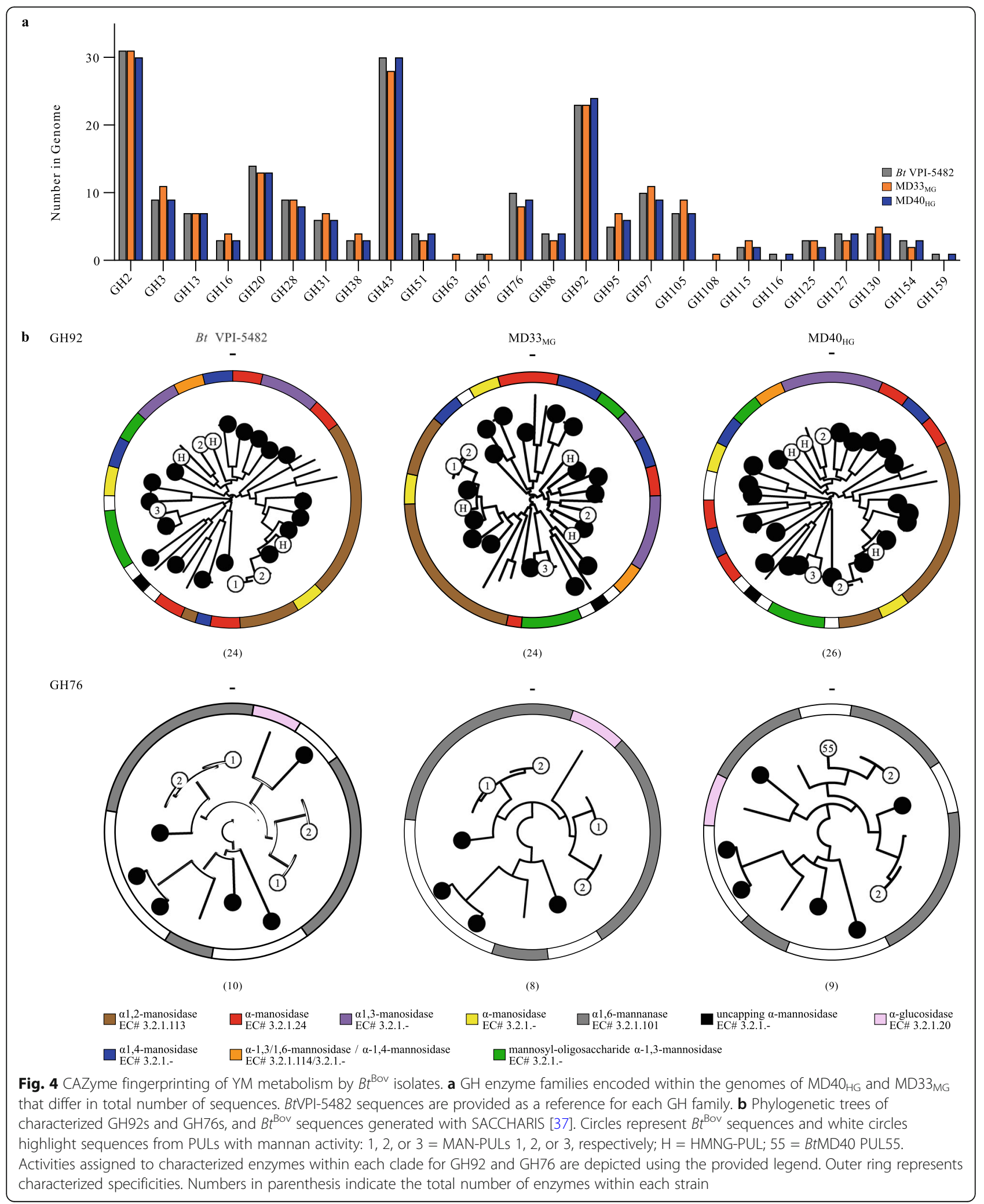

and 3 and HMNG-PUL from $B t$ VPI-5482, MD33 $3_{\mathrm{MG}}$, and $\mathrm{MD} 40_{\mathrm{HG}}$ revealed that proteins cluster into functional clades (Fig. 5a). Furthermore, when the SusC-like and SusD-like transport proteins from each MD strain were aligned with BT_3788 (Fig. 5b) and BT_3789 (Fig. 5c) of BtVPI-5482, respectively, the proteins partitioned 
exclusively into clades associated with either the MG or HG phenotype. This result is in contrast with the $16 \mathrm{~S}$ rRNA (Fig. 2a) and whole-genome (Supplementary Table 2) alignments, which showed no correlation with growth profiles. Interestingly, this pattern does not exist for MAN-PUL1 or MAN-PUL3 as the SusC-like proteins in these pathways are highly conserved (Fig. 5a), suggesting that syntenic conservation may not always reflect sequence-function relationships.

To study how transporters affect YM uptake, different combinations of sus $C / D$-like genes were excised from the BtVPI-5482 MAN-PULs. Three mutant strains were produced: a MAN-PUL2 susC-like and susD-like gene knock-out strain $(\triangle \mathrm{MP} 2 s u s C D)$, a MAN-PUL1 and 3 susC-like and susD-like deletion mutant ( $\triangle \mathrm{MP} 1 /$ $3 s u s C D$ ), and a strain with all three sets of susC-like and susD-like genes deleted $(\triangle \mathrm{MP} 1 / 2 / 3$ sus $C D)$. Because MAN-PUL1 is absent in every HG except BtVPI-5482, we can conclude it has no effect on YM transport efficiency and that the $\triangle \mathrm{MP} 1 / 3$ sus $C D$ mutant essentially operates as a $B t^{\text {Bov }}$ MAN-PUL3 susCD knock-out strain. The mutants, along with BtVPI-5482, were grown on YM-MM to assess how the loss of transport complexes impacted growth on YM (Fig. 5d). Surprisingly, the mutants retained an identical growth profile to the wildtype, with the exception of the triple knock-out mutant $(\triangle \mathrm{MP} 1 / 2 / 3$ sus $C D)$, which displayed no growth. Furthermore, when the mutants were incubated with FLA-YM, to study the impact on uptake rates, they displayed identical rates to the wild-type, with only the triple deletion mutant having a complete loss of FLA-YM import (Fig. $5 \mathrm{e}, \mathrm{f})$. These results demonstrated that the SusC-like/ SusD-like proteins from MAN-PUL2 and MAN-PUL3 in $B t$ VPI-5482 are functionally redundant. Although the absence of genetic tools prevented the investigation of the interplay between the MD33 $3_{\mathrm{MG}}$ transporters, the sequence divergence existing between MAN-PUL2 SusC/ $\mathrm{D} / \mathrm{E}$-like proteins from MD33 ${ }_{\mathrm{MG}}$ and $\mathrm{MD} 40_{\mathrm{HG}}$ (Supplementary Table 4) suggested that the dichotomous MG and HG growth phenotypes may result from differential transport through these complexes.

\section{Comparative analysis of gene expression between $B t^{\text {Bov }}$ growth phenotypes}

RNA-seq was performed on BtVPI-5482, MD33 $3_{\mathrm{MG}}$, and MD40 ${ }_{\mathrm{HG}}$ cultured on either mannose or YM to explore differential patterns in expression of the enzymes and transporters in the MAN-PULs and identify any distally

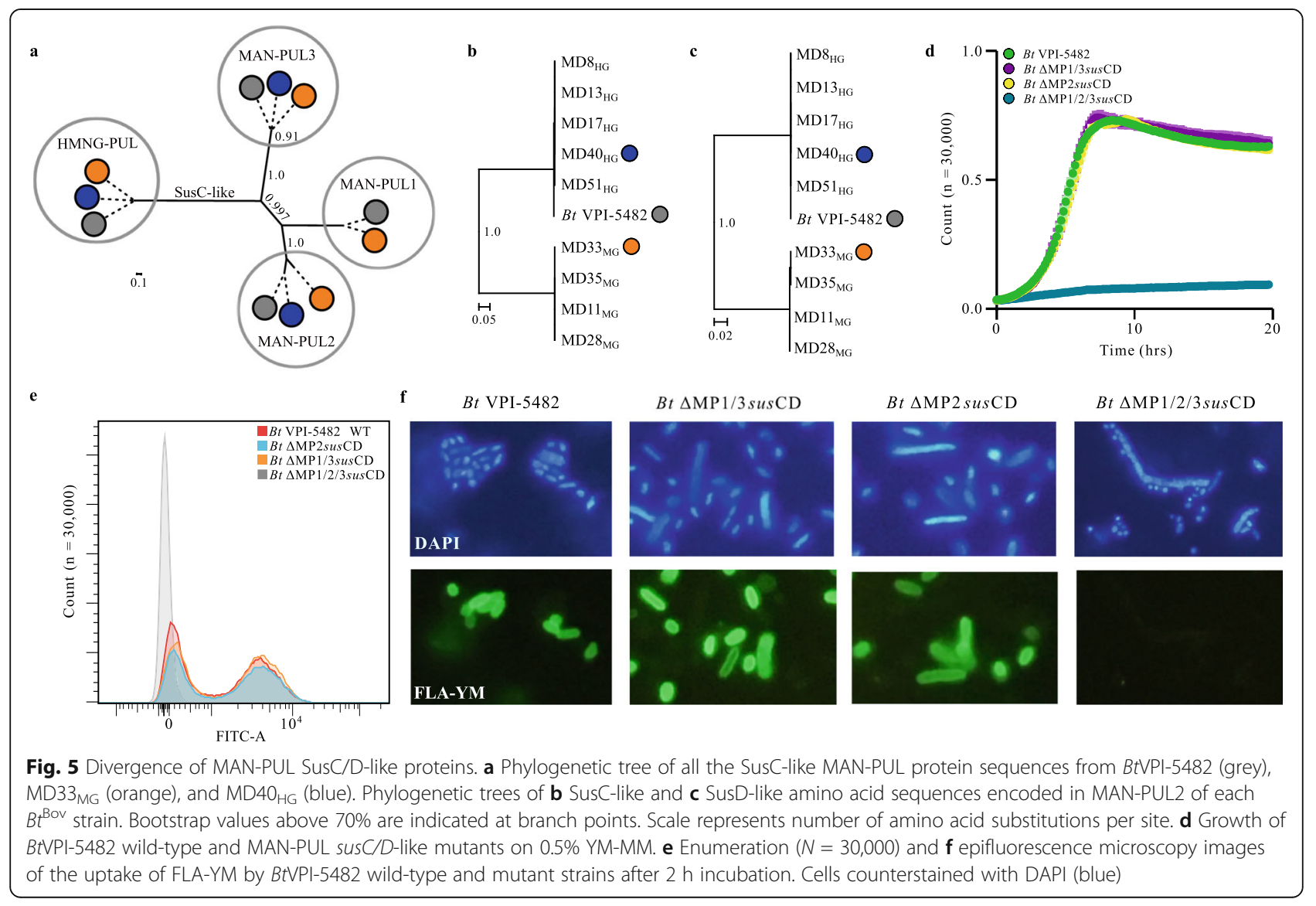


expressed genes. MAN-PUL2 and MAN-PUL3 pathways were activated in all three bacteria, and MAN-PUL1 was activated in BtVPI-5482 and MD33 MG (Fig. 6a, Supplementary Fig. 6) consistent with previous reports for BtVPI-5482 [18] (see Supplementary Discussion). To confirm that gene expression was representative of protein production, a C-Myc tag was fused to the Cterminal of the MAN-PUL2 SusD-like protein (BT_ 3789) in the chromosome of BtVPI-5482. Extracellular display of BT_3789 was demonstrated using antibodies directed at C-Myc when this bacterium was cultured on YM but not mannose (Supplementary Fig. 7).
The TPM values for every homologous gene transcript

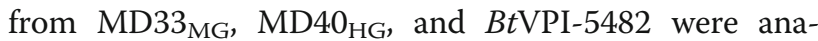
lyzed. Surprisingly, the sus-like genes (BT_3788 and BT_ 3789 ) and the surface enzyme transcripts (BT_3792, BT 2623, and BT_3858) of MD33 ${ }_{M G}$ consistently displayed significantly higher expression levels than the HG strains (Fig. 6b, Supplementary Fig. 6b). These values ranged between $6.2-\log _{2}$ and $7.7-\log _{2}$, suggesting that the expression level of gene products involved in outer membrane processing and intracellular transport is negatively correlated with growth proficiency on YM. The only example of an enzyme that is expressed at a significantly

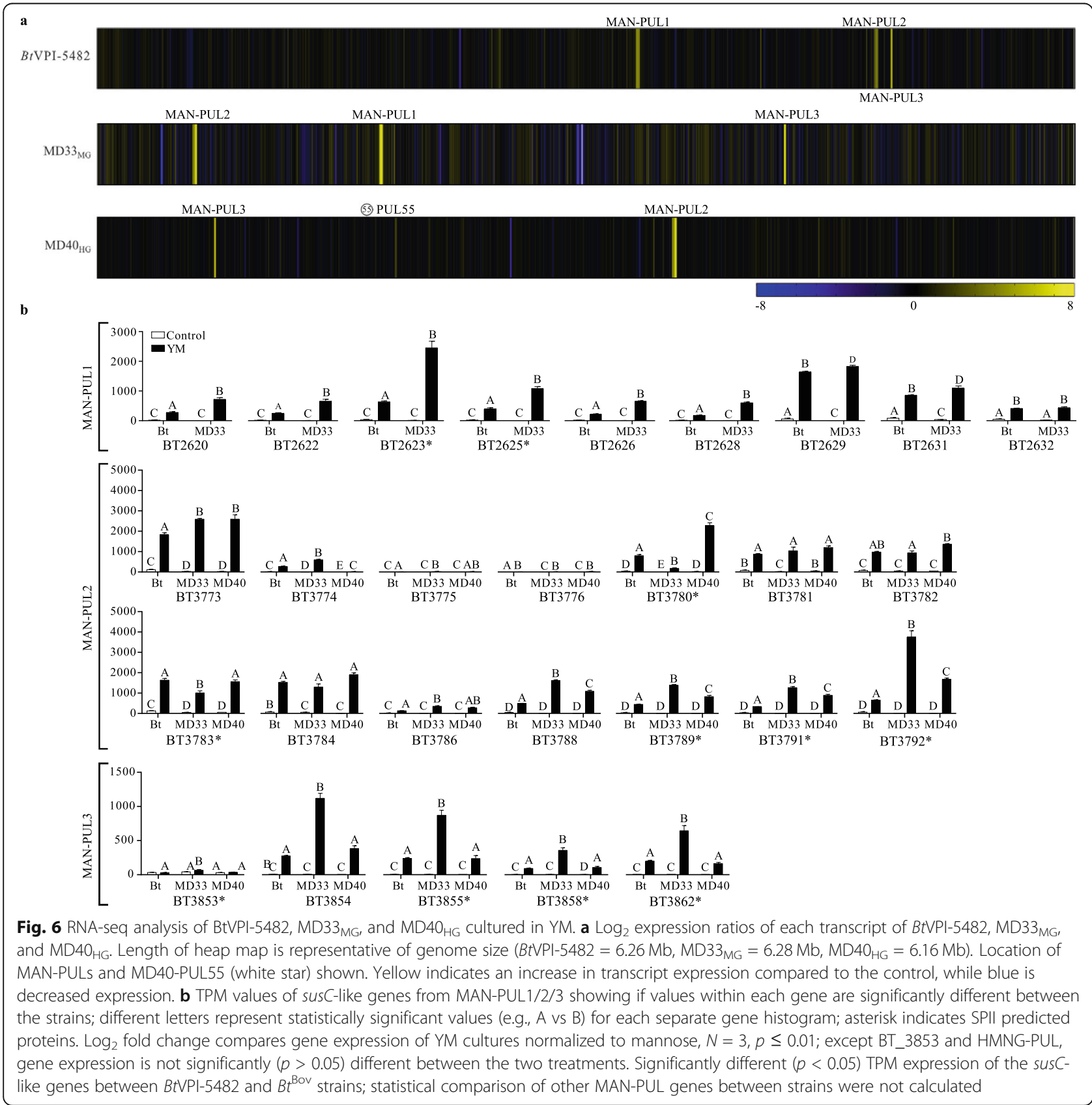


higher level in the $\mathrm{MD}_{40} 0_{\mathrm{HG}}$ strain was BT_3780 (12.5fold higher than MD33 ${ }_{\mathrm{MG}}$ ), which encodes a GH130 that is active on $\beta-1,2$-mannosides [40].

\section{Differences in YM hydrolysis and import}

The enzymatic processing of YM (amount of YM products, extent of YM utilization, and total free mannose present in the post-growth supernatants) by each culture was analyzed using a combination of methods. Thin layer chromatography (TLC) (Fig. 7a) revealed that there was no detectable free mannooligosaccharides or mannose in the supernatant of the YM-MM negative control. Consistent with this observation, the BtMAN-PUL1/2/3 deletion mutant $(\triangle M A N-P U L 1 / 2 / 3)$ did not grow on $\mathrm{YM}$ and was unable to release products into the medium. BtVPI-5482 and each of the HGs generated a similar product profile, with a noticeable loss of YM signal and faint detection of oligosaccharides and mannose. In contrast, the post-growth media of MGs contained more mannose and had residual YM (Fig. 7a). Gas chromatography-mass spectrometry determined that the quantity of total mannosides (YM and oligosaccharides) in the supernatant was 1.48 and 1.40-fold higher for MD33 $_{\text {MG }}(1.36 \pm 0.29)$ than BtVPI-5482 (0.92 \pm 0.04$)$ and $\mathrm{MD} 4 \mathrm{H}_{\mathrm{HG}}(0.97 \pm 0.05)$, respectively (Fig. $\left.7 \mathrm{~b}\right)$. Furthermore, post-growth $B t \mathrm{VPI}-5482$ and $\mathrm{MD} 40_{\mathrm{HG}}$ cultures, but not MD33 ${ }_{\mathrm{MG}}$, showed $(p<0.05)$ lower total mannose concentration in the media relative to the YMMM negative control (Fig. 7b). This suggests that, consistent with their higher growth densities (Fig. 2b) and thin layer chromatography, BtVPI-5482 and MD40 consume more YM.

To determine if surface $\alpha$-mannanases generate different product profiles, cultures of $B t \mathrm{VPI}-5482, \mathrm{MD} 33_{\mathrm{MG}}$, and $\mathrm{MD} 40_{\mathrm{HG}}$ were incubated with FLA-YM and the products were analyzed by fluorescence-coupled high- performance liquid chromatography. In BtVPI-5482 and MD40 ${ }_{\mathrm{HG}}$, there was preferential hydrolysis of large FLAYM products ( $>10 \mathrm{kDa}, \sim 55$-mer), which was accompanied by a relative accumulation of lower molecular weight ( $<4 \mathrm{kDa} ; \sim 22$-mer) hydrolysis products (Fig. 7c, Supplementary Fig. 8).

\section{Discussion}

The gut microbiome plays an integral role in digestion and nutrient acquisition. Improved understanding of the functional potential encoded within members of the microbiota is still required to define metabolic abilities and microbial-prebiotic interactions. Next-generation physiology approaches represent promising strategies to rapidly assign cellular phenotypes and can consolidate genomic predictions [29]. By combining phenotypic and sequencing approaches, we have conducted a highresolution study of differential YM utilization by isolated bovine-associated bacterial strains. In liquid culture, the isolates displayed one of two growth patterns: MG or HG; trends that were independent of taxonomic relationships (Fig. 2a, b, Supplementary Table 2). This showed that closely related Bacteroides spp. have evolved different foraging strategies for the same substrate. FLAPS were successfully used to visualize (Fig. 2c) and quantify the accumulation (Fig. 2d) and uptake rate (Fig. 2e) of YM products in bacterial cells, confirming that HGs use a selfish mode of metabolism on this substrate, as previously reported for $B t \mathrm{VPI}-5482$ [14, 31]. In contrast, the MG strains consumed less YM and released mannose into the medium (Fig. 7a, b), suggesting that MGs are less adept at YM catabolism and display some properties consistent with distributive metabolism (Figs. 2b and $7 \mathrm{c})$.

Comparative genomics revealed genotypes with high synteny across genomes and MAN-PUL pathways, with

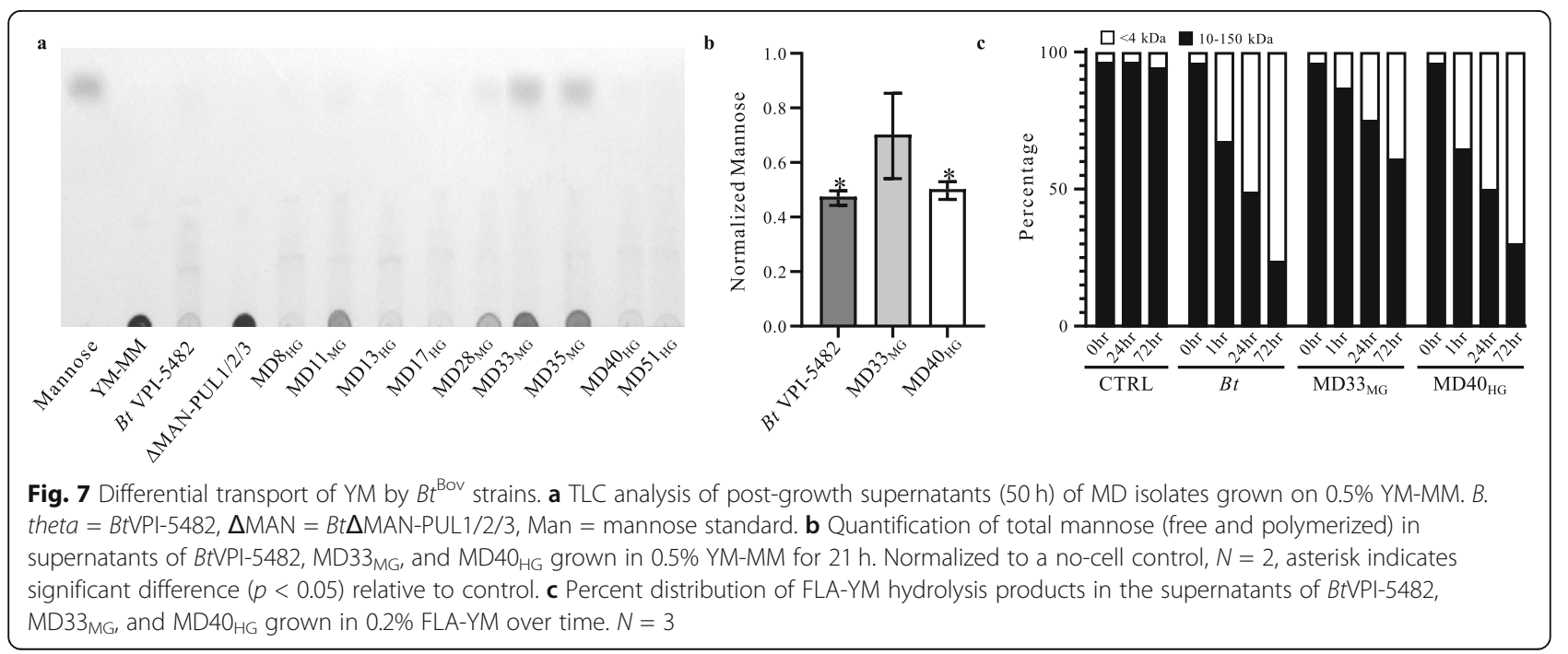


few exceptions. Perhaps the most interesting genetic anomaly is the sequence variability of the MAN-PUL2 SusC/D-like proteins, which elegantly branch into two clades coinciding with the HG and MG growth phenotype (Fig. 5b, c), as well as differential rates and total levels of FLA-PS uptake (Fig. 2c-e, Supplementary Fig. 2b). Previously, it was shown that the amino acid homology of a SusD-like protein involved in utilization of two different fructans was low between two strains of $B$. theta, despite their taxonomic similarity [41], highlighting that syntenic genes within PULs can evolve independently. Here we report that SusC/D-like amino acid sequences from the major PUL involved in metabolism of YM correlate with differential utilization of a common substrate (Fig. 5b, c). MD40 ${ }_{\mathrm{HG}}$ likely has a more efficient transport process (Fig. 7), as suggested by the following results: there is no perceived difference in the structures of surface enzymes encoded within the MAN-PULs (Supplementary Fig. 4), the outer surface endo- $\alpha$ mannanases are expressed at lower levels in HGs (Fig. $6 \mathrm{~b})$, the addition of exogenous endo-GH76s to MG growth cultures did not augment MG growth (Supplementary Fig. 5), and the higher growth and faster disappearance of large $\mathrm{YM}$ products in $\mathrm{MD} 40_{\mathrm{HG}}$ cultures (Fig. 7). Whether this is the direct result of higher transporter efficiency in $\mathrm{MD} 40_{\mathrm{HG}}$ or indirect result from impoverished transport leading to product inhibition of surface enzymes in MD33 ${ }_{\mathrm{MG}}$ is unclear. Intriguingly, deletion of MAN-PUL1/3 susC/D or the MAN-PUL2 sus $C / D$ did not impede growth of BtVPI-5482 on YM or uptake of YM (Fig. 5d-f), suggesting SusC/D-like pairs in MAN-PUL2 and 3 are functionally redundant in HGs. Based upon sequence identity (Supplementary Table 4), MGs possess one compromised SusC/D/E complex (MAN-PUL2) and one high-performing SusC/D complex (MAN-PUL3), which are regulated differently between the strains. In MD33 ${ }_{\mathrm{MG}}$, the MAN-PUL3 susClike gene (bt3854 homolog) is expressed at a level similar to the MAN-PUL2 susC-like gene (bt3788 homolog), and at a level 2.9-fold higher than its homologous gene in $\mathrm{MD}_{40} \mathrm{HG}$ (Fig. 6b). Higher expression of outer surface proteins in $\mathrm{MD}^{3} 3_{\mathrm{MG}}$ is a consistent pattern (Fig. 6b). Despite the higher expression levels of the MAN-PUL3 SusC/D-like complex in MD33 $3_{\mathrm{MG}}$, and the ability of the MAN-PUL3 SusC/D-like complex to compensate for deletion of the MAN-PUL2 transporter in BtVPI-5482 (Fig. 5d, e), the MAN-PUL3 SusC/D-like complex in $\mathrm{MD}_{3} 3_{\mathrm{MG}}$ is unable to rescue the MG growth phenotype of the representative strain. Thus, the SusC/D-like complexes in MAN-PUL2 and MAN-PUL3 appear to compete for substrates and the inefficiencies of transport ascribed to the MAN-PUL2 complex are related to its ability to transport, but not recruit, YM substrates. Further biochemical and structural studies of the MAN-
PUL2 SusC/D-like proteins are warranted to tease apart these results.

The "Nutrient Niche Hypothesis" [42] suggests that metabolic abilities are determined by the creation and filling of ecological nutrient niches. In theory, these relationships could be in response to the introduction of a new dietary glycan (i.e., prebiotic), resulting in the selection for or adaptation of a bacterium with the metabolic capacity to consume it. In this study, the MG and HG phenotypes represent a variation on this theme, as two closely related populations ( $>98 \%$ identity) adapted to the colonization of a common host (Supplementary Fig. 3) display different (Fig. 2b, Supplementary Fig. 1), yet reproducible (Fig. 3a) and inducible (Fig. 3b-d) foraging behaviors on the same substrate. These findings raise several unsolved questions related to the existence and persistence of MGs, and potentially other glycan foragers that are less adept at substrate utilization, in the rumen. If HGs have a superior capacity for YM metabolism, why are MGs not eliminated by competitive exclusion? And if MGs have restricted abilities to digest YM and/or transport YM products (Fig. 7), why are these PULs not selected against and excised from the genome? The existence of multiple metabolic phenotypes suggests that ecological selection factors may be responsible. Firstly, Bacteroides spp. are generalists with the capacity to utilize a wide variety of substrates available in the diet of their hosts and glycan responses are prioritized in Bacteroides spp. [43, 44]. MGs may possess a different substrate hierarchy than HGs and, correspondingly, display more prowess for consuming chemically distinct glycans. Alternative substrate priorities would reduce the competitive burden on MGs when provided with complex diets. In this regard, the acquisition of new CAZymes or PULs that endow a microorganism with an ability to consume new substrates has been hypothesized to occur by horizontal gene transfer and is linked to spatial and dietary habits $[20,28]$. YM from S. cerevisiae (Supplementary Fig. 1a) and S. pombe (Supplementary Fig. 1b) were the substrates used in this study and showcased that HGs are not consistently superior when it comes to glycan utilization. Further investigation into the ability of MGs and HGs to utilize other substrates is warranted to identify additional variability in substrate utilization and preference. Secondly, feeding strategies, such as distributive metabolism, may foster beneficial syntrophic relationships at multiple levels within a community [45, 46]. The generation of public goods [13] by MGs provides nutrients to species that are incapable of digesting YM. This event would increase the richness of the community and, potentially, result in the generation of additional secondary metabolites that benefit the lifestyle of MGs. Furthermore, it has been shown that both the concentrations and complexity of available substrate cause 
differential selection of distributive or selfish foraging strategies [47-49].

Comparison of the MD40 ${ }_{\mathrm{HG}}$ and MD33 $3_{\mathrm{MG}}$ CAZomes confirmed that there are many $\mathrm{GH}$ families, encoding different enzyme specificities that vary in number (Fig. 4a). Closer inspection of GH3 and GH16, two polyspecific $\mathrm{GH}$ families active on $\beta$-linkages, revealed CAZyme updates within a PUL in MD33 $3_{\text {MG }}$ (Fig. 8). This suggests that the acquisition of a putative $\beta$-glucan metabolic pathway, and potentially others, may provide a colonization advantage for MD33 $3_{\mathrm{MG}}$ despite its weakened potential to metabolize YM. Recently, $\beta$-glucan utilization pathways were shown to have independently evolving genes that result in the expansion of protein specificity and glycan targets [50]. Thus, clustered mutations or differential acquisition of genes in PULs could unlock previously inaccessible nutrient niches. Conversely, however, there is the risk of impeding nutrient acquisition, as exhibited by the restricted $\beta$-glucan utilization of polyspecific proteins in Bacteroides fluxus [50] and, potentially, the inefficiencies of YM uptake governed by transporter specificity or efficiency. Further investigation of total CAZome function and transporter selectivity and efficiency encoded within genomes at the strain level will reveal how microorganisms living in partnership or competition within complex ecosystems tune their metabolic responses to complex dietary landscapes. Coupling "omics" methods and functional methods, such as FLA-PS, will help usher in a new frontier for the assignment of metabolic traits to bacterial populations within microecological food webs

\section{Materials and methods}

Direct visualization of YM metabolism in rumen communities and cell identification by FISH

Rumen samples were collected from two cannulated cows fed a diet rich in barley grain. The rumen samples were filtered through cheesecloth under $\mathrm{CO}_{2}$ gas. Subsamples were taken, flash frozen, and stored at $-80^{\circ} \mathrm{C}$ until genomic extractions could be completed. The rest of the sample was transferred into an anaerobic chamber (atmosphere: $85 \% \mathrm{~N}_{2}, 10 \% \mathrm{CO}_{2}, 5 \% \mathrm{H}_{2}$, at $37^{\circ} \mathrm{C}$ ) and filtered through a $100-\mu \mathrm{m}$ pore size nylon net filter (Millipore, USA). The filtered samples from each cow were
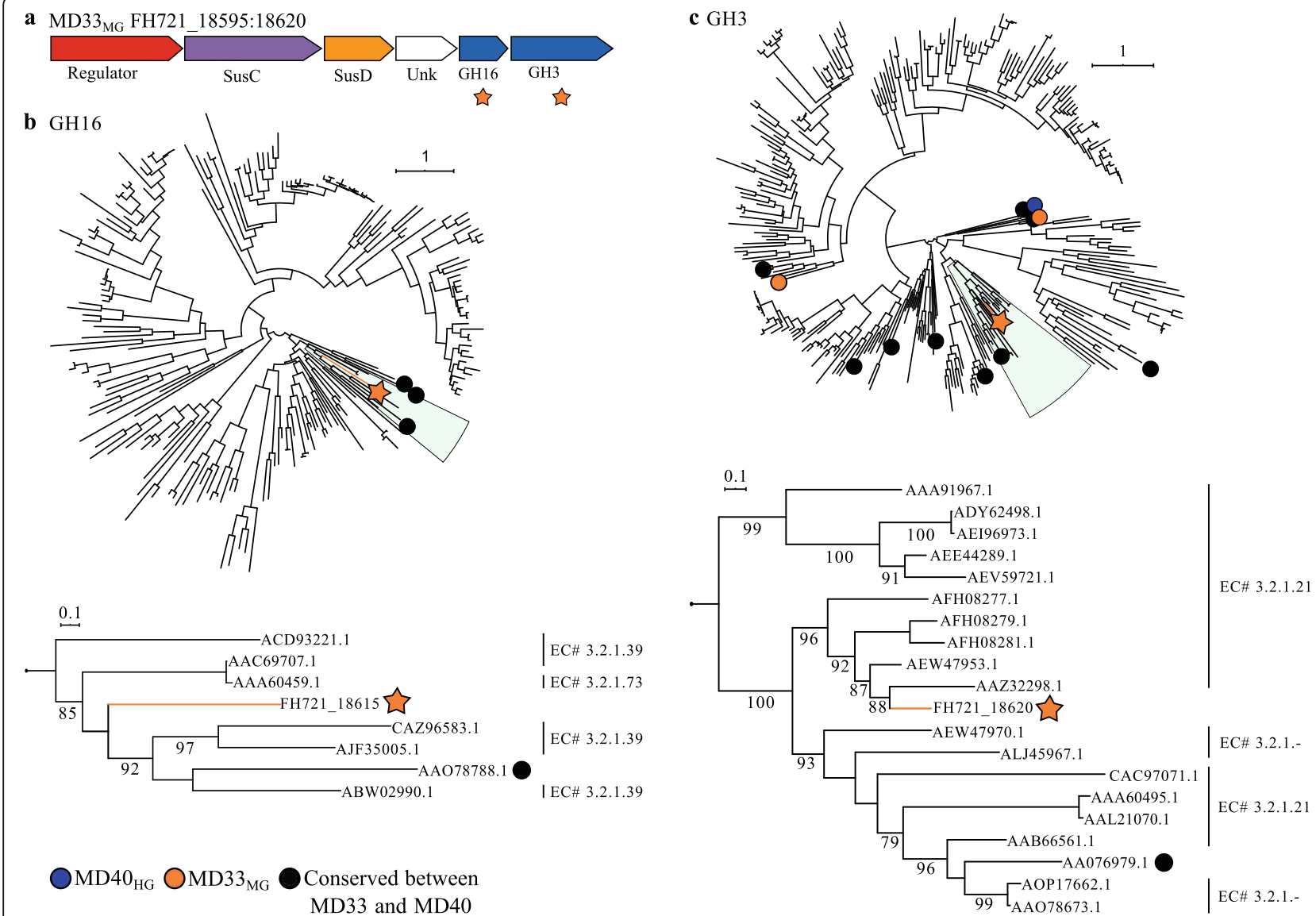

$\mathrm{MD} 40_{\mathrm{HG}} \mathrm{OMD} 33_{\mathrm{MG}}$

Conserved between MD33 and MD40

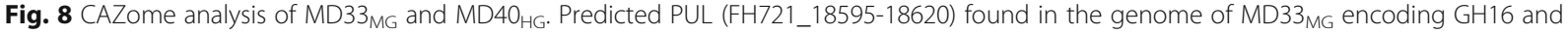
$\mathrm{GH} 3$ enzymes, which are not found in the MD40 $\mathrm{HG}_{\text {g }}$ genome. Phylogenetic trees of GH16 (top) and GH3 (bottom) families in MD33 $\mathrm{MG}$ (orange) and $\mathrm{MD}_{4} \mathrm{H}_{\mathrm{HG}}$ (blue) genomes. (Left) Expanded clades with the unique GH16 and GH3 CAZymes are shown with known EC numbers 
then aliquoted into three tubes. One tube was immediately fixed with $1 \%$ formaldehyde (FA) for $1 \mathrm{~h}$ at room temperature as the $0 \mathrm{~h}$ control. The other tubes were incubated with $20 \mu \mathrm{L}$ FLA-YM for a final concentration of $3.1 \mathrm{nM}$ and fixed with FA after 1 day and 3 days. Immediately after fixation, all samples were filtered through a $47 \mathrm{~mm}(0.2-\mu \mathrm{m}$ pore size) polycarbonate filter (Millipore), using a $0.45-\mu \mathrm{m}$ cellulose acetate support filter (Millipore) and a gentle vacuum of $<200$ mbar. After drying, the filters were stored at $-20^{\circ} \mathrm{C}$.

Total cell counts were determined by staining with 4 , 6-diamidino-2-phenylindole (DAPI) and visualizing on a Leica DMRX epifluorescence microscope (Leica, Germany). The number of FLA-YM stained cells was determined by enumerating cells which had a positive DAPI and FLA-YM signal (excitation at 405-nm and 488-nm wavelengths, respectively). For FISH, oligonucleotide probe CF968 targeting Bacteroidetes (5'GGTAAGGTTCCTCGCGTA-3') [33] was used, which was covalently labeled with four ATTO594 fluorochromes by Biomers (Konstanz, Germany). FISH was performed with slight alterations to the protocol of Manz et al. [51]. The hybridization buffer contained 900 $\mathrm{mM} \mathrm{NaCl}, 20 \mathrm{mM}$ TRIS-HCl (pH 7.5), $0.02 \%$ sodium dodecyl sulfate, $10 \%$ dextran sulfate $(\mathrm{w} / \mathrm{v})$, and $1 \%(\mathrm{w} / \mathrm{v})$ blocking reagent (Boehringer, Germany) with a formamide concentration of $55 \%$. Hybridizations were carried out at $35^{\circ} \mathrm{C}$ in a humidity chamber overnight, with a subsequent 15 -min wash in a buffer containing $10 \mathrm{mM}$ $\mathrm{NaCl}, 20 \mathrm{mM}$ TRIS-HCl (pH 7.5), 5 nM EDTA (pH 8), and $0.01 \%$ sodium dodecyl sulfate at $37{ }^{\circ} \mathrm{C}$. After FISH, the abundance of Bacteroidetes as well as Bacteroidetes showing FLA-YM uptake was enumerated using a Leica DMRX epifluorescence microscope.

DNA from the frozen rumen samples was extracted using the Qiagen DNeasy PowerSoil Kit, and samples were sent to McGill GenomeQuebec for Illumina MiSeq PE250 16S rRNA metagenomics sequencing. The 16S rRNA sequences were merged and quality trimmed using the BBTools [52] software and subsequently classified using the standard settings of the SILVAngs pipeline using the SSU rRNA seed of the SILVA database release 132 [53]. All analysis and plotting of the microbial diversity data were done using RStudio version 3.6.3 using the Vegan package $[54,55]$

\section{Isolation of bovine-adapted mannan degraders}

Bovine rumen and fecal samples were collected for in vitro batch culture experiments. Ruminal and fecal inoculants from cattle were enriched anaerobically (atmosphere: $\left.85 \% \mathrm{~N}_{2}, 10 \% \mathrm{CO}_{2}, 5 \% \mathrm{H}_{2}\right)$ at $37^{\circ} \mathrm{C}$ with one of the following substrates: Bio-Mos ${ }^{\bullet}(1 \% \mathrm{w} / \mathrm{v})$, corn distillers' grains $(1 \% \mathrm{w} / \mathrm{v})$, or YM $(1 \% \mathrm{w} / \mathrm{v})$. Bacteria were isolated from the enriched batch cultures by streaking onto nutrient-restricted media supplemented with $0.5 \%$ YM to select for YM-degraders (supplementary methods). In total, 50 YM-degrading bacterial isolates were characterized for their propensity to metabolize YM. Nine of these isolates were selected for detailed analysis in this study.

\section{Growth profiling of bovine isolates}

Bovine isolates, wild-type $B t \mathrm{VPI}-5482$, and a mutant $B t$ strain lacking MAN-PULs 1 , 2, and 3 (MMAN-PUL1/2/ 3) [14] were cultured anaerobically (atmosphere: $85 \%$ $\mathrm{N}_{2}, 10 \% \mathrm{CO}_{2}, 5 \% \mathrm{H}_{2}$ ) at $37^{\circ} \mathrm{C}$ overnight in tryptoneyeast-glucose (TYG) medium (supplementary methods). All incubations were performed in an anaerobic chamber at $37{ }^{\circ} \mathrm{C}$. The overnight cultures $\left(\mathrm{OD}_{600}\right.$ 1.0-1.4) were diluted to an $\mathrm{OD}_{600}$ of 0.05 in $2 \mathrm{X}$ Bacteroides minimal medium (MM), pH 7.2 (supplementary methods). Wells of a 96-well microtiter plates (Falcon) were filled with $100 \mu \mathrm{L}$ of sterilized 1\% (w/v) YM (Sigma, St. Louis, USA; M7504) or mannose along with $100 \mu \mathrm{L}$ inoculant $(n=4)$. Negative control wells consisted of $100 \mu \mathrm{L} 2 \mathrm{X}$ MM combined with $100 \mu \mathrm{L} \mathrm{1 \%} \mathrm{(w/v)} \mathrm{of} \mathrm{YM} \mathrm{or} \mathrm{mannose}$ and were used to normalize growth curves. One hundred microliters of bacterial suspension was inoculated to get starting $\mathrm{OD}_{600} \sim 0.025$. Plates were sealed with polyurethane Breathe-Easy gas-permeable membranes (Sigma; Z390059). Absorbance $(600 \mathrm{~nm})$ of each well was measured with a Biotek Eon microplate reader and recorded on Biotek Gen 5 software every $10 \mathrm{~min}$ for $50 \mathrm{~h}$. Mean $( \pm$ standard deviation) of each condition $(n=4)$ was visualized using GraphPad Prism 6. Two replicates of each strain were also cultured on YM extracted from the cell wall of $S$. pombe (supplementary methods).

Post-growth cultures were harvested and centrifuged. Supernatants were taken and $6 \mu \mathrm{L}$ was ran on a silica sheet in 2:1:1 (butanol to $\mathrm{d}_{2} \mathrm{H} 2 \mathrm{O}$ to acetic acid) running buffer. The plate was dried at ambient temperature and stained with orcinol (diluted to $1 \%$ in a solution of $70: 3$ ethanol to sulfuric acid). Once the plate was dry, it was activated in an oven at $120^{\circ} \mathrm{C}$ and imaged using a gel doc XR image system (Bio-Rad).

\section{Genome sequencing, assembly, and annotation of $B t^{B o v}$ strains}

The 16S rRNA of 50 bovine bacterial isolates was sequenced to determine taxonomic classification using the universal primers $27 \mathrm{~F}$ and 1492R (supplementary methods). Based on growth profiles $\left(\mathrm{OD}_{600}>0.4\right)$ and 16S rRNA sequences, nine isolates were chosen for whole-genome sequencing using Illumina MiSeq PE150 bp. Genomes were assembled using SPAdes de novo assembly [35]. The K-mer value in SPAdes was chosen from (21, 33, 55, and 77 defaults for $150 \mathrm{bp}$ reads). Quality reporting of the assemblies was done using Quast 
[56]. SPAdes assembly N50s, largest contigs, and number of contigs are shown in Supplementary Table 2. Genomes were uploaded to the NCBI submission portal and annotated using the NCBI Prokaryotic Annotation Pipeline. Isolate contigs were blasted against the reference genome BtVPI-5482 for MAN-PUL1/2/3 and the HMNG-PUL using NCBI BLAST (2.7.1) [34]. SPAdes contig assemblies were aligned with the JSpeciesWS reference $B t \mathrm{VPI}-5482$ genomes to calculate average nucleotide identity based on BLAST+ (ANIb) [36].

\section{Production of BtVPI-5482 MAN-PUL mutants}

Flanking regions $(\sim 750 \mathrm{bp})$ of the susC/D-like genes from each MAN-PUL were PCR amplified, stitched together, and ligated into pExchange-tdk (pEx-tdk). The plasmids were transformed into E. coli strain S17-1 $\lambda$ pir, which were donor cells used to conjugate the plasmids into the BtVPI-5482 $\Delta$ PUL75 $\Delta$ tdk recipient strain to delete the sus-like gene pairs [57]. Mutants with the MAN-PUL1 Sus genes deleted $(\triangle \mathrm{MP} 1$ sus $C D)$ were then conjugated with $E$. coli cells containing a plasmid with the flanking regions for the MAN-PUL3 Sus genes to create a dual mutant $(\triangle \mathrm{MP} 1 / 3$ sus $C D)$. The dual mutant was then conjugated with $E$. coli cells that contained a plasmid with the MAN-PUL2 Sus flanks to produce a triple mutant $(\triangle \mathrm{MP} 1 / 2 / 3$ sus $C D)$. Plasmids and mutants were sequenced at each step of this process.

The three knock-out strains, along with BtVPI-5482 wild-type, were grown on $0.5 \%$ YM-MM as described above. In addition, the strains were incubated with FLAYM and sampled at $0 \mathrm{~h}, 1 \mathrm{~h}, 1$ day, and 3 days. These samples were fixed and stored at $4{ }^{\circ} \mathrm{C}$ until analyzed by flow cytometry and epifluorescence microscopy (see below).

\section{PUL delineation and comparative CAZomics}

Isolate contigs were processed through EMBOSS GetORF [58] to determine open reading frames; these data were run through the dbCAN [59] HMMscan to identify CAZyme sequences. CAZyme sequences were then analyzed by SACCHARIS [37] (Sequence Analysis and Clustering of CarboHydrate Active enzymes for Rapid Informed prediction of Specificity). User CAZyme sequences were trimmed to their catalytic domain with dbCAN [59], aligned with MUSCLE [60], and fitted to a phylogenetic tree using ProtTest3 [61] to find the appropriate amino acid replacement model. RAxML [62] or FastTree [63] was used to generate the final tree. GHs from families 38, 76, 92, 99, and 125 identified in the genomes of the MD isolates were analyzed by SACCHARI S. Phylogenetic trees were developed using FastTree, and Newick file outputs were viewed and plotted using ITOL (doi.org/10.1093/nar/gkz239).
RNA-seq: assembly, quantitation, and comparative analysis

RNA from $B t \mathrm{VPI}-5482$, MD33 ${ }_{\mathrm{MG}}$, and $\mathrm{MD} 40_{\mathrm{HG}}$ grown in $0.5 \%$ mannose or YM (see supplementary methods) was extracted and purified using a GeneJET RNA Purification kit (Thermo Scientific) within 1 week of storage at $-80^{\circ} \mathrm{C}$. RNA was sent to Génome Québec for Illumina HiSeq 4000 PE100bp sequencing. Using Geneious v11.1.2 [64], each set of reads was mapped to their previously assembled genomic sequence or, in the case of $B t \mathrm{VPI}-5482$, to the genomic sequence from the NCBI database (NC_004663). Expression levels were calculated as transcript expression (transcript per kb per million; TPM) for each growth treatment. Ambiguously mapped reads were counted as partial matches. The Geneious DESeq2 [65] plugin was used to compare the expression levels between the two treatments, producing $\log _{2}$ expression ratios and $p$ values.

Generalized linear mixed models in SAS PROC GLIM MIX (SAS 9.4, SAS Institute, Cary, NC, USA) were used to estimate statistically significant $(p<0.05)$ differences of TPM means (least squares-means) for the MANPUL1/2/3 susC-like genes of each bacterial strain. Based on the Bayesian information criterion (BIC) of the generalized linear mixed models, the response was modeled using the log-normal distribution. The expression of gene transcripts was the dependent variable in models with two independent fixed factors: bacterial strain (i.e., BtVPI-5482, MD33 $3_{\mathrm{MG}}$, or $\mathrm{MD} 40_{\mathrm{HG}}$ ) and media treatment (i.e., YM or mannose). Mixed models of variance heterogeneity were selected based on the BIC. For the studied transcripts, the variance of expression was heterogeneous for the experimental treatments, bacteria, or their interaction. The statistical significance of the interaction between the TPM values of MAN-PUL genes for each bacterial strain and the media treatment was determined using an $F$ test. Bonferroni's method was used for multiple comparisons (Supplementary Table 3).

\section{Production of SusD-like protein C-myc fusion B. theta strain}

The C-myc epitope (EQKLISEEDL) was fused to the Cterminal domain of the MAN-PUL2 SusD-like protein (BT_3789) with a linker sequence (STSTST) between the SusD-like nucleotide sequence and the C-myc sequence BtVPI-5482 $\Delta$ tdk $\Delta$ pul75 (control), and BtVPI$5482 \Delta$ tdk $\Delta$ pul75 SusD-like C-myc fusion mutant was inoculated in TYG and cultured as described above. The cells were centrifuged and resuspended in $1 \mathrm{~mL} 2 \mathrm{X}$ MM. One hundred microliters of the resuspension was inoculated into $0.5 \%$ YM-MM and incubated for $4 \mathrm{~h}$ at $37^{\circ} \mathrm{C}$. The cells were then centrifuged and washed three times in phosphate-buffered saline (PBS) pH 7.4 (PBS; $137 \mathrm{mM}$ $\left.\mathrm{NaCl}, \quad 2.7 \mathrm{mM} \quad \mathrm{KCl}, \quad 10 \mathrm{mM} \quad \mathrm{Na}_{2} \mathrm{HPO}_{4}\right)$, before 
resuspension in $2 \mathrm{~mL} 2 \mathrm{X}$ MM. Two hundred twenty-five microliters of the resuspended cells was added to $1.5 \mathrm{~mL}$ $0.2 \%$ FLA-YM or YM-MM and incubated for $3 \mathrm{~h}$ at $37^{\circ} \mathrm{C}$. One hundred microliters of each culture was collected and fixed in $1 \%$ formaldehyde for $1 \mathrm{~h}$ at room temperature. The samples were then incubated with 1 : 2500 rabbit IgG anti-C-myc polyclonal antibody (ThermoFisher \#PA1-981) for $1 \mathrm{~h}$ at room temperature. The samples were then washed four times in PBS and resuspended in 1:2500 goat anti-rabbit DyLight $650 \mathrm{~nm}$ secondary antibody (ThermoFisher \#84546) for $1 \mathrm{~h}$ at room temperature. The samples were then washed and stored in PBS until further analysis.

\section{Sequence comparison and modeling of SusC/D/E-like proteins}

MUSCLE was used to align MAN-PUL2 and 3 SusClike, SusD-like (SGBPA), and SusE-like (SGBPB) amino acid sequences of each isolate and calculate percent identity (Supplementary Table 4). The 16S rRNA gene and MAN-PUL2 SusC-like and SusD-like amino acid phylogenetic trees were generated using the maximum likelihood method and Tamura-Nei model [66]. Evolutionary analyses were performed by MEGA X [67]. Trees with the highest log likelihood are shown in Fig. $4 \mathrm{~b}$ and c.

\section{Generation of FLA-YM conjugates}

A previously defined protocol $[30,68]$ was used to generate fluorescently labeled YM (FLA-YM), with slight variations (supplementary methods).

\section{Visualization of FLA-YM uptake by strains of $B t^{\text {Bov }}$} Wild-type $B t$ VPI-5482, Bt $\Delta$ MAN-PUL1/2/3, and rumen isolates MD33 ${ }_{\mathrm{MG}}$ and $\mathrm{MD} 40_{\mathrm{HG}}$ were inoculated in TYG and grown as described above. Cells were harvested at $\mathrm{OD}_{600} \sim 1.0$ and centrifuged $(4700 \times g)$ for $5 \mathrm{~min}$, the supernatant was removed, and pellets resuspended in 2 $\mathrm{mL} 2 \mathrm{X} \mathrm{MM}$ for the first two washes. After the third centrifugation, pellets were resuspended in $2 \mathrm{~mL}$ MM with $0.5 \% \mathrm{YM}$ (BtVPI-5482, MD33 ${ }_{\mathrm{MG}}$, and $\mathrm{MD} 40_{\mathrm{HG}}$ ) or $0.5 \%$ glucose + YM (Bt $\Delta$ MAN-PUL1/2/3) as the sole carbon source (not conjugated to FLA). After $\sim 18$-h incubation, cultures were centrifuged and washed three times in PBS, with the final resuspension in $2 \mathrm{~mL} 2 \mathrm{X}$ MM. Three hundred microliters of the resuspended pellet was aliquoted into $0.2 \%$ unlabeled YM or FLA-YM. Twenty microliters of the $2 \mathrm{X}$ MM resuspension was used as the 0$\mathrm{h}$ time point, as the cells were not exposed to FLA-YM. Forty microliters aliquots of each condition were taken at time points: $5 \mathrm{~min}, 1 \mathrm{~h}$, and $24 \mathrm{~h}$. The cells were centrifuged ( $10 \mathrm{~min} ; 2300 \times \mathrm{g}$ ), and the pellet was fixed in $1 \%$ formaldehyde (FA; Sigma; F8775) in PBS, at $4{ }^{\circ} \mathrm{C}$ for 18 $24 \mathrm{~h}$. The fixed cells were centrifuged ( $10 \mathrm{~min} ; 2300 \times g$ ) and washed in $1 \mathrm{X}$ PBS. The samples were centrifuged and stored at $4{ }^{\circ} \mathrm{C}$ in the dark until visualized by SR-SIM (supplementary methods).

\section{Quantification of the rate of FLA-YM uptake by $B t^{\text {Bov }}$ isolates}

BtVPI-5482, MD33 ${ }_{\mathrm{MG}}$, and $\mathrm{MD} 40_{\mathrm{HG}}$ were grown in TYG and prepared as described above. After $24 \mathrm{~h}$ of incubation, cultures were placed into $2 \mathrm{~mL} 0.5 \%$ YM. Cells were harvested in exponential phase $\left(\mathrm{OD}_{600}\right.$ 0.6-1.0), centrifuged $(10 \mathrm{~min} ; 2300 \times g)$, and resuspended in $2 \mathrm{~mL}$ 2X MM. Three hundred microliters of this suspension was added to $1 \mathrm{~mL} 2 \mathrm{X}$ MM. Then $20 \mu \mathrm{L}$ of each culture was aliquoted into $1 \mathrm{~mL} 1 \% \mathrm{FA}$ and used as the T0 time point. Into the remaining $280 \mu \mathrm{L}, 0.2 \%$ FLA-YM and $150 \mathrm{ng} / \mathrm{mL}$ fluoresceinamine (FLA) or YM was added and subsamples of $40 \mu \mathrm{L}$ were taken at $5,10,15,20,30$, and $60 \mathrm{~min}$ and $2,4,8$, and $24 \mathrm{~h}$. The subsamples were centrifuged $(10 \mathrm{~min}, 2300 \times g)$, and the cell pellets were fixed in $1 \% \mathrm{FA}$ in $1 \mathrm{X} \mathrm{PBS}$, at $4{ }^{\circ} \mathrm{C}$ for $18-24 \mathrm{~h}$. The fixed cells were centrifuged (10 min; $2300 \times g$ ) and resuspended in $1 \mathrm{ml} 1 \mathrm{X}$ PBS and stored at $4{ }^{\circ} \mathrm{C}$ in the dark.

Cell fluorescence due to FLA or FLA-YM uptake was quantified in all samples using an Accuri C6 flow cytometer (BD Accuri Cytometers). The 8-peak and 6-peak validation bead suspensions (Spherotech, IL, USA) were used as internal references. All samples were measured under laser excitation at $488 \mathrm{~nm}$ from a blue-green laser, and the green fluorescence was collected in the FL1 channel $(530 \pm 30 \mathrm{~nm})$. Using the medium as a background, an electric threshold of $17,000 \mathrm{FSC}-\mathrm{H}$ was set to reduce the background noise. All measurements were done at a slow flow rate and a total of 10,000 (FLA-YM) or 5000 (YM and FLA) events per sample were acquired. Bacteria were detected from the signature plot of SSC-H vs green fluorescence (FL1-H). The FCM output was analyzed using FlowJo v10-4-2 (Tree Star, USA). The FCM files were imported into FlowJo, and both the total population (all events) and main population (automated gating through event density) were determined. For each population (total and main), sample statistics (counts, mean fluorescence, and the standard deviation) were determined from the raw FL1-H data. The results were exported and analyzed using Welch's $t$ tests in R studio using the packages Vegan and Rioja $[55,69]$ to determine statistical difference between the control (YM and FLA) and FLA-YM incubation within each strain and between the FLA-YM incubation of each strain.

\section{Quantification of mannose in minimal medium using GC- MS}

Cell culture medium after incubation in 1\% YM-MM was collected after $24 \mathrm{~h}$ and centrifuged $(4700 \times g$ for 15 mins), and the supernatant was passed through a syringe 
filter $(0.2 \mu \mathrm{m}$ cellulose acetate membrane, VWR $)$. The filtrate was kept frozen for $48 \mathrm{~h}$ at $-20{ }^{\circ} \mathrm{C}$ and then thawed and centrifuged $(3000 \times g, 30 \mathrm{~min})$ at room temperature. Concentration of mannose in the resulting supernatant was tested based on our previous report [70], with some modifications to cope with the relatively large amount of starting carbohydrate material and the presence of minimum medium. One milliliter of the supernatant was evaporated to dryness under a gentle flow of nitrogen. The residue was suspended and magnetically stirred in $3.5 \mathrm{~mL}$ of $6 \mathrm{M} \mathrm{TFA}$ at $100^{\circ} \mathrm{C}$ for $6 \mathrm{~h}$ with headspace filled with nitrogen, followed by addition of internal standard myo-inositol $(0.4 \mathrm{mg}$ dissolved in $0.5 \mathrm{~mL}$ of water) and evaporation to dryness. Monosaccharides were reduced by magnetic stirring overnight in $10 \mathrm{mg}$ of NaBD4 (99\% D, Alfa Aesar) dissolved in $2 \mathrm{~mL}$ of $1 \mathrm{M}$ ammonium oxide solution, followed by quenching excess reductant with acetic acid and evaporating to dryness. Boric acid was removed by evaporation to dryness five times in $3 \mathrm{~mL}$ of $10 \%(\mathrm{v} / \mathrm{v})$ acetic acid in methanol followed by five times in $3 \mathrm{~mL}$ of absolute methanol. The residue was suspended in $4 \mathrm{~mL}$ of acetic anhydride, followed by magnetic stirring at $100^{\circ} \mathrm{C}$ for 2 $\mathrm{h}$ with headspace filled with nitrogen, cooling to room temperature, and evaporation to dryness. The derivatives were purified by partitioning with water and dichloromethane, recovered by collecting and evaporating to dryness the organic phase after three changes of water, and re-dissolved and diluted in ethyl acetate for analysis on an Agilent 7890A-5977B GC-MS system (Agilent Technologies, Inc., CA, USA). Sample solution $(1 \mu \mathrm{L})$ was splitless-injected to the system, and optimal analyte separation was achieved on a medium polarity SP2380 column $(30 \mathrm{~m} \times 0.25 \mathrm{~mm} \times 0.20 \mu \mathrm{m}$, Sigma-Aldrich $)$ with a constant helium flow of $0.8 \mathrm{~mL} / \mathrm{min}$ and with oven temperature programmed to start at $55^{\circ} \mathrm{C}$ (hold $1 \mathrm{~min}$ ) followed by increasing at $30^{\circ} \mathrm{C} / \mathrm{min}$ to $120^{\circ} \mathrm{C}$ then at $12^{\circ} \mathrm{C} / \mathrm{min}$ to $255^{\circ} \mathrm{C}$ (hold $20 \mathrm{~min}$ ). Two separate experiments were conducted for each sample. Mannose concentration was calculated based on calibration curve established from a series of mannose standard solution containing internal standard.

\section{Measurement of YM hydrolysis}

Samples from BtVPI-5482, MD33 ${ }_{\mathrm{MG}}$, and $\mathrm{MD} 40_{\mathrm{HG}}$ cultures in $0.2 \%$ FLA-YM (as above) and a no-cell negative control were filtered through $0.2-\mu \mathrm{m}$ cellulose acetate membrane syringe filter (VWR). The filtrates were flash frozen and stored at $-80^{\circ} \mathrm{C}$ until analysis. Samples were analyzed as described in Arnosti 2003 [68]; in brief, samples were injected onto two columns of Sephadex G50 and G75 gel linked in series, with the column effluent passing through a Hitachi fluorescence detector set to excitation and emission wavelengths of 490 and $530 \mathrm{~nm}$, respectively. The columns were standardized using FITC dextran standards $(150 \mathrm{kDa}, 70 \mathrm{kDa}, 40 \mathrm{kDA}, 10 \mathrm{kDa}, 4$ $\mathrm{kDa}$, FITC-glucose, and free fluorophore; Sigma), so the fraction of polysaccharide eluting in each molecular weight class at each time point could be calculated.

\section{Supplementary Information}

The online version contains supplementary material available at https://doi. org/10.1186/s40168-020-00975-x.

Additional file 1. Supplementary figures

Additional file 2. Supplementary materials

\section{Acknowledgements}

We wish to thank Sherif Ghobrial and Chad Lloyd for the assistance with the FLA-YM hydrolysis measurements, and Elizabeth Lowe, Newcastle University, for the kind gift of the MAN-PUL1/2/3 KO mutant.

\section{Authors' contributions}

LK assisted with the rumen extractions and performed the rumen incubations with FLA-YM, selective bacterial growth profiling and TLC analysis, RNA sequencing, production of FLA-YM, FGC-bacterial incubations, construction of the SusD-C-myc mutant and evaluation of production, and extraction of S. pombe YM; prepared figures; and wrote manuscript. GR conducted the epifluorescence microscopy and SR-SIM analysis, FISH analysis, cell enumeration of rumen samples, flow cytometry sorting and analysis, and statistical analysis and assisted with figure and manuscript writing and preparation. JPT performed comparative genome and protein sequence analysis and assisted with figure preparation. DRJ assisted with comparative genome and protein analysis. $\mathrm{JHH}$ helped conceive of the study, interpreted the data, and assisted in the preparation of the manuscript. ADS performed isolations of rumen bacteria and $16 \mathrm{~S}$ rRNA sequencing. TDS conducted the statistical analysis of RNA-seq data. CAr assisted with the generation of FLA-PS, analysis FLA-PS products, and preparation of the manuscript. LJ performed the rumen collections and assisted with the bacterial isolations. TWA assisted with the rumen extractions and preparation of the manuscript. CAm assisted with the RNA-seq analysis and figure generation. DT assisted with the CAZyme fingerprinting and maintenance of the SACCHARIS pipeline. RA helped conceive of the study and assisted with the SR-SIM microscopy and preparation of the manuscript. TAM assisted with the animal study, rumen extractions, and preparation of the manuscript. DPY assisted with the data analysis and preparation of the manuscript. DWA helped conceive of the study, secured the funding, designed the study, performed the data analysis, and assisted with figure and manuscript preparation. The authors read and approved the final manuscript.

\section{Funding}

This work was supported by funding from the Beef and Cattle Research Council awarded to DWA (Grant: FDE.13.15 \& FDE.14.17). JHH, GR, and RA were supported by the Max Planck Society. RA and JHH acknowledge the support by the Deutsche Forschungsgemeinschaft (DFG) in the framework of the research unit FOR2406 "Proteogenomics of Marine Polysaccharide Utilization (POMPU)" by grants AM 73/9-1 and HE 7217/1-1. GR has received funding from the European Union's Horizon 2020 research and innovation program under the Marie Sklodowska-Curie grant agreement No. 840804. CAr was supported by NSF OCE-1736772, DE-SC0013887, and DE-SC0019012.

\section{Availability of data and materials}

The datasets generated and analyzed during the current study are available in the NCBI repository in the whole-genome sequencing BioProject No: PRJNA546576 (BioSample Accession Numbers: MD8 ${ }_{\text {HG }}$, SAMN11961934; MD11 $_{M G}$ SAMN11961935; MD13 ${ }_{\mathrm{HG}}$ SAMN11961936; MD17 SG $_{\mathrm{H},}$ SAMN11961937; MD28 ${ }_{M G}$, SAMN11961938; MD33 ${ }_{M G}$, SAMN11961939;

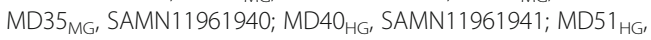
SAMN11961942), and RNA sequencing BioProject No: PRJNA658335 (BioSample Accession Numbers: BtVPI-5482, SAMN15866569; MD33 ${ }_{M G}$ SAMN15866570; MD40 ${ }_{\mathrm{HG}}$ SAMN15866571). The read mapping of RNA-seq data was done using the assembled genomic sequences from the BioProject 
or to the genomic sequence of BtVPI-5482 found in the NCBI database (NC_004663).

\section{Competing interests}

The authors declare that they have no competing interests.

\section{Author details}

${ }^{1}$ Lethbridge Research and Development Centre, Agriculture and Agri-Food Canada, 5403-1st Avenue South, Lethbridge, Alberta T1J 4B1, Canada. ${ }^{2}$ Department of Biological Sciences, University of Lethbridge, Lethbridge, Alberta T1K 3M4, Canada. ${ }^{3}$ Max Planck Institute for Marine Microbiology, 28359 Bremen, Germany. ${ }^{4}$ Center for Marine Environmental Sciences, University of Bremen (MARUM), 28359 Bremen, Germany. ${ }^{5}$ Department of Marine Sciences, University of North Carolina, Chapel Hill 27599-3300, NC, USA

Received: 26 June 2020 Accepted: 7 December 2020

Published online: 22 January 2021

\section{References}

1. Weimer PJ. Redundancy, resilience, and host specificity of the ruminal microbiota: implications for engineering improved ruminal fermentations. Front Microbiol. 2015;6:296.

2. Henderson G, Cox F, Ganesh S, Jonker A, Young W. Global Rumen Census C, Janssen PH: Rumen microbial community composition varies with diet and host, but a core microbiome is found across a wide geographical range. Sci Rep. 2015;5:14567.

3. Stewart RD, Auffret MD, Warr A, Wiser AH, Press MO, Langford KW, Liachko I, Snelling TJ, Dewhurst RJ, Walker AW, et al. Assembly of 913 microbial genomes from metagenomic sequencing of the cow rumen. Nat Commun. 2018;9:870.

4. Huws SA, Creevey CJ, Oyama LB, Mizrahi I, Denman SE, Popova M, MuñozTamayo R, Forano E, Waters SM, Hess M, et al. Addressing global ruminant agricultural challenges through understanding the rumen microbiome: past, present, and future. Front Microbiol. 2018;9:2161.

5. Lima J, Auffret MD, Stewart RD, Dewhurst RJ, Duthie C-A, Snelling TJ, Walker AW, Freeman TC, Watson M, Roehe R. Identification of rumen microbial genes involved in pathways linked to appetite, growth, and feed conversion efficiency in cattle. Front Genet. 2019;10:701.

6. Uyeno $Y$, Shigemori S, Shimosato T. Effect of probiotics/prebiotics on cattle health and productivity. Microbes Environ. 2015;30:126-32.

7. Foley MH, Cockburn DW, Koropatkin NM. The Sus operon: a model system for starch uptake by the human gut Bacteroidetes. Cell Mol Life Sci. 2016;73: 2603-17.

8. Grondin JM, Tamura K, Dejean G, Abbott DW, Brumer H. Polysaccharide utilization loci: fueling microbial communities. J Bacteriol. 2017;199:e00860

9. Anderson KL, Salyers AA. Biochemical evidence that starch breakdown by Bacteroides thetaiotaomicron involves outer membrane starch-binding sites and periplasmic starch-degrading enzymes. J Bacteriol. 1989;171:3192-8.

10. Kruger K, Chafee M, Ben Francis T, Glavina Del Rio T, Becher D, Schweder T, Amann $\mathrm{Rl}$, Teeling $\mathrm{H}$. In marine Bacteroidetes the bulk of glycan degradation during algae blooms is mediated by few clades using a restricted set of genes. ISME J. 2019;13:2800-16.

11. Martens EC, Kelly AG, Tauzin AS, Brumer H. The devil lies in the details: how variations in polysaccharide fine-structure impact the physiology and evolution of gut microbes. J Mol Biol. 2014;426:3851-65.

12. Teeling H, Fuchs BM, Becher D, Klockow C, Gardebrecht A, Bennke CM, Kassabgy M, Huang S, Mann AJ, Waldmann J, et al. Substrate-controlled succession of marine bacterioplankton populations induced by a phytoplankton bloom. Science. 2012;336:608-11.

13. Rakoff-Nahoum S, Coyne MJ, Comstock LE. An ecological network of polysaccharide utilization among human intestinal symbionts. Curr Biol. 2014;24:40-9.

14. Cuskin F, Lowe EC, Temple MJ, Zhu Y, Cameron EA, Pudlo NA, Porter NT, Urs K, Thompson AJ, Cartmell A, et al. Human gut Bacteroidetes can utilize yeast mannan through a selfish mechanism. Nature. 2015;517:165-9.

15. Abbott DW, Martens EC, Gilbert HJ, Cuskin F, Lowe EC. Coevolution of yeast mannan digestion: Convergence of the civilized human diet, distal gut microbiome, and host immunity. Gut Microbes. 2015;6:334-9.
16. Terrapon N, Lombard V, Drula E, Lapebie P, Al-Masaudi S, Gilbert HJ, Henrissat B. PULDB: the expanded database of polysaccharide utilization Loci. Nucleic Acids Res. 2018;46:D677-83.

17. Larsbrink J, Rogers TE, Hemsworth GR, McKee LS, Tauzin AS, Spadiut O, Klinter S, Pudlo NA, Urs K, Koropatkin NM, et al. A discrete genetic locus confers xyloglucan metabolism in select human gut Bacteroidetes. Nature. 2014;506:498-502.

18. Martens EC, Lowe EC, Chiang H, Pudlo NA, Wu M, McNulty NP, Abbott DW, Henrissat B, Gilbert HJ, Bolam DN, Gordon J. Recognition and degradation of plant cell wall polysaccharides by two human gut symbionts. PLoS Biol. 2011;9:e1001221.

19. Hehemann JH, Kelly AG, Pudlo NA, Martens EC, Boraston AB. Bacteria of the human gut microbiome catabolize red seaweed glycans with carbohydrateactive enzyme updates from extrinsic microbes. Proc Natl Acad Sci U S A. 2012;109:19786-91.

20. Pluvinage B, Grondin JM, Amundsen C, Klassen L, Moote PE, Xiao Y, Thomas D, Pudlo NA, Anele A, Martens EC, et al. Molecular basis of an agarose metabolic pathway acquired by a human intestinal symbiont. Nat Commun. 2018:9:1043.

21. Davies G, Henrissat B. Structures and mechanisms of glycosyl hydrolases. Structure. 1995;3:853-9.

22. Lombard V, Golaconda Ramulu H, Drula E, Coutinho PM, Henrissat B. The carbohydrate-active enzymes database (CAZy) in 2013. Nucleic Acids Res. 2014:42:D490-5.

23. Finck DN, Ribeiro FRB, Burdick NC, Parr SL, Carroll JA, Young TR, Bernhard BC, Corley JR, Estefan AG, Rathmann RJ, Johnson BJ. Yeast supplementation alters the performance and health status of receiving cattle. Prof Anim Sci. 2014:30:333-41.

24. Miguel JC, Rodriguez-Zas SL, Pettigrew JE. Efficacy of a mannan oligosaccharide (Bio-Mos ${ }^{\oplus}$ ) for improving nursery pig performance. J Swine Health Prod. 2004;12:296-307.

25. Wohlt JE, Corcione TT, Zajac PK. Effect of yeast on feed intake and performance of cows fed diets based on corn silage during early lactation. $J$ Dairy Sci. 1998:81:1345-52.

26. Gregg KJ, Zandberg WF, Hehemann JH, Whitworth GE, Deng L, Vocadlo DJ, Boraston AB. Analysis of a new family of widely distributed metalindependent alpha-mannosidases provides unique insight into the processing of N-linked glycans. J Biol Chem. 2011;286:15586-96.

27. Zhu Y, Suits MD, Thompson AJ, Chavan S, Dinev Z, Dumon C, Smith N, Moremen KW, Xiang Y, Siriwardena A, et al. Mechanistic insights into a Ca2+-dependent family of alpha-mannosidases in a human gut symbiont. Nat Chem Biol. 2010;6:125-32.

28. Hehemann JH, Correc G, Barbeyron T, Helbert W, Czjzek M, Michel G. Transfer of carbohydrate-active enzymes from marine bacteria to Japanese gut microbiota. Nature. 2010;464:908-12.

29. Hatzenpichler R, Krukenberg V, Spietz RL, Jay ZJ. Next-generation physiology approaches to study microbiome function at single cell level. Nat Rev Microbiol. 2020;18:241-56.

30. Reintjes G, Arnosti C, Fuchs BM, Amann R. An alternative polysaccharide uptake mechanism of marine bacteria. ISME J. 2017;11:1640-50.

31. Hehemann JH, Reintjes G, Klassen L, Smith AD, Ndeh D, Arnosti C, Amann R, Abbott DW. Single cell fluorescence imaging of glycan uptake by intestinal bacteria. ISME J. 2019;13:1883-9.

32. Tao J, McCourt C, Sultana H, Nelson C, Driver J, Hackmann TJ. Use of a fluorescent analog of glucose (2-NBDG) to identify uncultured rumen bacteria that take up glucose. Appl Environ Microbiol. 2019;85:e03018.

33. Acinas SG, Ferrera I, Sarmento H, Díez-Vives C, Forn I, Ruiz-González C, Cornejo-Castillo FM, Salazar G, Gasol JM. Validation of a new catalysed reporter deposition-fluorescence in situ hybridization probe for the accurate quantification of marine Bacteroidetes populations. Environ Microbiol. 2015;17:3557-69.

34. Altschul SF, Gish W, Miller W, Myers EW, Lipman DJ. Basic local alignment search tool. J Mol Biol. 1990;215:403-10.

35. Bankevich A, Nurk S, Antipov D, Gurevich AA, Dvorkin M, Kulikov AS, Lesin VM, Nikolenko SI, Pham S, Prjibelski AD, et al. SPAdes: a new genome assembly algorithm and its applications to single-cell sequencing. J Comput Biol. 2012;19:455-77.

36. Richter M, Rosselló-Móra R, Oliver Glöckner F, Peplies J. JSpeciesWS: a web server for prokaryotic species circumscription based on pairwise genome comparison. Bioinformatics (Oxford, England). 2016;32:929-31.

37. Jones DR, Thomas D, Alger N, Ghavidel A, Inglis GD, Abbott DW. SACCHARI $S$ : an automated pipeline to streamline discovery of carbohydrate active 
enzyme activities within polyspecific families and de novo sequence datasets. Biotechnol Biofuels. 2018;11:27.

38. Ndeh D, Rogowski A, Cartmell A, Luis AS, Basle A, Gray J, Venditto I, Briggs J, Zhang X, Labourel A, et al. Complex pectin metabolism by gut bacteria reveals novel catalytic functions. Nature. 2017;544:65-70.

39. Jones DR, Xing X, Tingley JP, Klassen L, King ML, Alexander TW, Abbott DW. Analysis of active site architecture and reaction product linkage chemistry reveals a conserved cleavage substrate within diverse yeast mannans for an endo-alpha-mannanase. J Mol Biol. 2020;432:1083-97.

40. Cuskin F, Basle A, Ladeveze S, Day AM, Gilbert HJ, Davies GJ, PotockiVeronese G, Lowe EC. The GH130 family of mannoside phosphorylases contains glycoside hydrolases that target beta-1,2-mannosidic linkages in Candida Mannan. J Biol Chem. 2015:290:25023-33.

41. Joglekar P, Sonnenburg ED, Higginbottom SK, Earle KA, Morland C, ShapiroWard S, Bolam DN, Sonnenburg JL. Genetic variation of the SusC/SusD homologs from a polysaccharide utilization locus underlies divergent fructan specificities and functional adaptation in Bacteroides thetaiotaomicron strains. mSphere. 2018;3:e00185-18.

42. Freter $\mathrm{R}$, Brickner $\mathrm{H}$, Botney $\mathrm{M}$, Cleven $\mathrm{D}$, Aranki A. Mechanisms that control bacterial populations in continuous-flow culture models of mouse large intestinal flora. Infect Immun. 1983:39:676-85.

43. Rogers TE, Pudlo NA, Koropatkin NM, Bell JS, Moya Balasch M, Jasker K, Martens EC. Dynamic responses of Bacteroides thetaiotaomicron during growth on glycan mixtures. Mol Microbiol. 2013;88:876-90.

44. Tuncil YE, Xiao Y, Porter NT, Reuhs BL, Martens EC, Hamaker BR. Reciprocal prioritization to dietary glycans by gut bacteria in a competitive environment promotes stable coexistence. mBio. 2017;8:e01068-17.

45. Koropatkin NM, Cameron EA, Martens EC. How glycan metabolism shapes the human gut microbiota. Nat Rev Microbiol. 2012;10:323-35.

46. Morris BE, Henneberger R, Huber H, Moissl-Eichinger C. Microbial syntrophy: interaction for the common good. FEMS Microbiol Rev. 2013;37:384-406.

47. Reintjes G, Fuchs BM, Scharfe M, Wiltshire KH, Amann R, Arnosti C. Short term changes in polysaccharide utilization mechanisms of marine bacterioplankton during a spring phytoplankton bloom. Environ Microbiol. 2020;22:1884-900.

48. Rogowski A, Briggs JA, Mortimer JC, Tryfona T, Terrapon N, Lowe EC, Baslé A, Morland C, Day AM, Zheng H, et al. Glycan complexity dictates microbial resource allocation in the large intestine. Nat Commun. 2015;6:7481.

49. Sarmento H, Morana C, Gasol JM. Bacterioplankton niche partitioning in the use of phytoplankton-derived dissolved organic carbon: quantity is more important than quality. ISME J. 2016;10:2582-92.

50. Déjean G, Tamura K, Cabrera A, Jain N, Pudlo NA, Pereira G, Viborg AH, Van Petegem F, Martens EC, Brumer H. Synergy between cell surface glycosidases and glycan-binding proteins dictates the utilization of specific beta(1,3)-glucans by human gut Bacteroides. mBio. 2020;11:e00095-20.

51. Manz W, Amann R, Ludwig W, Wagner M, Schleifer K-H. Phylogenetic oligodeoxynucleotide probes for the major subclasses of proteobacteria: problems and solutions. Syst Appl Microbiol. 1992;15:593-600.

52. Bushnell B, Rood J, Singer E. BBMerge - Accurate paired shotgun read merging via overlap. PLoS One. 2017;12:e0185056.

53. Quast C, Pruesse E, Yilmaz P, Gerken J, Schweer T, Yarza P, Peplies J, Glöckner FO. The SILVA ribosomal RNA gene database project: improved data processing and web-based tools. Nucleic Acids Res. 2013;41:D590-6.

54. Team R. RStudio: integrated development for R. Boston: RStudio. PBC; 2020.

55. Oksanen J, Kindt R, Legendre P, O'Hara B, Stevens MHH, Oksanen MJ, Suggests M. The vegan package. Comm Ecol Package. 2007;10:719.

56. Gurevich A, Saveliev V, Vyahhi N, Tesler G. QUAST: quality assessment tool for genome assemblies. Bioinformatics. 2013;29:1072-5.

57. Jones DR, Smith MB, McLean R, Grondin JM, Amundsen CR, Inglis GD, Selinger B, Abbott DW. Engineering dual-glycan responsive expression systems for tunable production of heterologous proteins in Bacteroides thetaiotaomicron. Sci Rep. 2019;9:17400.

58. Rice P, Longden I, Bleasby A. EMBOSS: the European Molecular Biology Open Software Suite. Trends Genet. 2000;16:276-7.

59. Yin Y, Mao X, Yang J, Chen X, Mao F. Xu Y: dbCAN: a web resource for automated carbohydrate-active enzyme annotation. Nucleic Acids Res. 2012; 40:W445-51.

60. Edgar RC. MUSCLE: multiple sequence alignment with high accuracy and high throughput. Nucleic Acids Res. 2004;32:1792-7.

61. Darriba D, Taboada GL, Doallo R, Posada D. ProtTest 3: fast selection of bestfit models of protein evolution. Bioinformatics. 2011;27:1164-5.
62. Stamatakis A. RAxML version 8: a tool for phylogenetic analysis and postanalysis of large phylogenies. Bioinformatics. 2014;30:1312-3.

63. Price MN, Dehal PS, Arkin AP. FastTree 2 - approximately maximumlikelihood trees for large alignments. PLoS One. 2010;5:e9490.

64. Kearse M, Moir R, Wilson A, Stones-Havas S, Cheung M, Sturrock S, Buxton S, Cooper A, Markowitz S, Duran C, et al. Geneious basic: an integrated and extendable desktop software platform for the organization and analysis of sequence data. Bioinformatics (Oxford, England). 2012;28:1647-9.

65. Love Ml, Huber W, Anders S. Moderated estimation of fold change and dispersion for RNA-seq data with DESeq2. Genome Biol. 2014;15:550.

66. Tamura K, Nei M. Estimation of the number of nucleotide substitutions in the control region of mitochondrial DNA in humans and chimpanzees. Mol Biol Evol. 1993;10:512-26.

67. Kumar S, Stecher G, Li M, Knyaz C, Tamura K. MEGA X: molecular evolutionary genetics analysis across computing platforms. Mol Biol Evol. 2018;35:1547-9.

68. Arnosti C. Fluorescent derivatization of polysaccharides and carbohydratecontaining biopolymers for measurement of enzyme activities in complex. J Chromatogr B. 2003;793:181-91.

69. Juggins $S$. rioja: analysis of quaternary science data; 2017.

70. MacMillan JL, Vicaretti SD, Noyovitz B, Xing X, Low KE, Inglis GD, Zaytsoff SJM, Boraston AB, Smith SP, Uwiera RRE, et al. Structural analysis of broiler chicken small intestinal mucin O-glycan modification by Clostridium perfringens. Poult Sci. 2019;98:5074-88.

\section{Publisher's Note}

Springer Nature remains neutral with regard to jurisdictional claims in published maps and institutional affiliations.

Ready to submit your research? Choose BMC and benefit from:

- fast, convenient online submission

- thorough peer review by experienced researchers in your field

- rapid publication on acceptance

- support for research data, including large and complex data types

- gold Open Access which fosters wider collaboration and increased citations

- maximum visibility for your research: over $100 \mathrm{M}$ website views per year

At BMC, research is always in progress.

Learn more biomedcentral.com/submissions 